\title{
Svecofennian intra-orogenic gabbroic magmatism: a case study from Turku, southwestern Finland
}

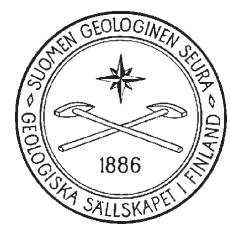

\author{
Jenni NeVAlainen ${ }^{1}$, MarkKu VÄIsÄnen $^{1 *}$, Yann Lahaye ${ }^{2}$, \\ Esa HeILImo ${ }^{3}$ and SÖREN FrÖJdö ${ }^{4}$ \\ ${ }^{1}$ Department of Geography and Geology, FI-20014, University of Turku, Finland \\ ${ }^{2}$ Geological Survey of Finland, P.O. Box 96, FI-02151 Espoo, Finland \\ ${ }^{3}$ Geological Survey of Finland, P.O. Box 1237, FI-70211 Kuopio, Finland \\ ${ }^{4}$ Institution of Geology, FI-20540 Ao Akademi University, Turku, Finland
}

\begin{abstract}
Using single-grain zircon U-Pb dating by LA-MC-ICPMS and whole-rock geochemistry, we have studied the Palaeoproterozoic gabbroic rocks from Moisio in southwest Finland. Three ages were obtained. The interpreted intrusion age is $\sim 1.86 \mathrm{Ga}$, which places it in the 1.87-1.84 Ga intra-orogenic period of southern Svecofennia. The other ages, 1.89 $\mathrm{Ga}$ and $\sim 1.83 \mathrm{Ga}$, are inferred to be inherited and metamorphic ages, respectively.

The K, LREE, LILE, Fe-, P-, Ti- and F-concentrations reveal two compositionally distinct groups: (i) an enriched monzogabbro group and (ii) a less enriched gabbro group. The composition of the monzogabbro group resembles the other intra-orogenic intrusions from southern Svecofennia, whereas the unrelated gabbro group is more comparable to the synorogenic rocks in the region. The magma source the monzogabbro experienced a subduction related carbonate metasomatism, induced by sediment subduction and subduction erosion. Evidently, the Moisio monzogabbro represent enriched, mantle derived magmatism in southern Svecofennia as a part of the intra-orogenic igneous activity. The intra-orogenic magmatism is considered to have conveyed considerable amounts of heat from the mantle into the crust contributing to subsequent lateorogenic high-grade metamorphism.
\end{abstract}

Keywords (GeoRef Thesaurus, AGI): magmatism, heat sources, metamorphism, monzogabbro, geochemistry, absolute age, U/Pb, zircon, Proterozoic, Paleoproterozoic, Moisio, Finland

* Corresponding author (e-mail: markku.vaisanen@utu.fi)

Editorial handling: Jussi S. Heinonen (jussi.s.heinonen@helsinki.fi) 


\section{Introduction}

Traditionally, the Svecofennian orogeny in southern Finland has been divided into the 1.89-1.86 Ga synorogenic, 1.84-1.80 Ga lateorogenic and 1.811.77 Ga postorogenic stages (Simonen, 1980; Nironen, 2005). The latest models and data outline two compressional orogenic stages with an intervening extensional period. These were coined as the earlier accretionary 1.89-1.87 Ga Fennian orogeny (synorogenic stage) and the later collisional 1.84-1.80 Ga Svecobaltic orogeny (lateorogenic stage) in the model proposed by Lahtinen et al. (2005). The intervening stage was designated as the intra-orogenic period. Sedimentary basins existed at the time shown by detrital zircons of intraorogenic age in quartzites in central Sweden and southern Finland (c. 1.87-1.84 Ga; Claesson et al., 1993; Lahtinen et al., 2002; Bergman et al., 2008) and ultra-mature lateritic paleosols found between the quartzites and the basement rocks below (Lahtinen \& Nironen, 2010). These findings and structural studies (Pajunen et al., 2008; Skyttä \& Mänttäri, 2008; Nironen \& Mänttäri, 2012) indicate that sedimentary basins were formed by extension of the Fennian crust. Associated intraorogenic magmatism was also expected (Lahtinen et al., 2005). Recently, single-grain zircon dating methods have revealed several 1.87-1.84 Ga mafic and felsic intrusions in southern Finland (Kurhila et al., 2005, 2010; Mänttäri et al., 2006; Pajunen, et al., 2008; Skyttä \& Mänttäri, 2008; Väisänen et al., 2012a). The mantle-derived intra-orogenic magmatism has been rather recently discovered and further research is required to better evaluate its role in the crustal evolution.

In this study mafic plutonic rocks from Moisio in the city of Turku, in SW Finland, are investigated (Fig. 1). Some of the analyses show geochemical similarities with the previously studied postcollisional (i.e. postorogenic) $1815 \pm 2 \mathrm{Ma}$ Urusvuori monzodiorite intrusion (Väisänen et al., 2000). However, the Moisio rocks are strongly deformed compared to the closely located Urusvuori intrusion, hampering the interpretation of age and tectonic setting of the Moisio rocks. We have therefore performed single-grain zircon U-Pb datings and whole-rock geochemical analyses from Moisio and compared the results with the data from the syn-, intra- and postorogenic intrusions in SW Finland in order to further understand the role of enriched mafic magmas for the Svecofennian evolution.

\section{Geological Setting}

In this study the following division for the magmatism in southern Svecofennia is used: preorogenic, synorogenic, intra-orogenic, lateorogenic and postorogenic (modified after Nironen, 2005; Fig. 1a). The preorogenic magmatism, defined as "island arc magmatism preceding onset of accretion or collision" (Nironen, 2005), comprises the 1.90-1.88 Ga volcanic rocks and their coeval plutonic magma chambers of the Häme belt and Uusimaa belts formed in volcanic arc settings (Väisänen et al. 2002; Ehlers et al., 2004; Kähkönen, 2005; Väisänen and Kirkland, 2008; Saalmann et al., 2009).

The Fennian orogeny accreted the Uusimaa and Häme belts against the Tampere belt and the Central Finland Granitoid Complex resulting in two main terranes, southern Svecofennia and central Svecofennia (Fig. 1a; Kähkönen, 2005; Lahtinen et al., 2005). The $1.87 \mathrm{Ga}$ synorogenic bimodal magmatism is coeval with the Fennian orogeny in southern Svecofennia (Väisänen et al., 2002; Lahtinen et al., 2005). The mafic magmatism is slightly enriched due to enrichment of the mantle source during the earlier subduction event whereas the felsic magmatism has a lower to mid-crustal source and shows adakite- and TTG-like character (Väisänen et al., 2012b).

The mafic intra-orogenic magmatism, dated at 1.865-1.85 Ga, shows elevated concentrations in $\mathrm{K}, \mathrm{Fe}, \mathrm{Ti}, \mathrm{P}, \mathrm{F}, \mathrm{LILE}$ (large ion lithophile elements) and LREE (light rare earth elements) and they are compositionally intermediate between the syn- and postorogenic magmatism in SW Finland (Väisänen et al., 2012a). Nironen (2011) describes intraorogenic volcanic rocks from southern Finland whose compositions are not as enriched as the 

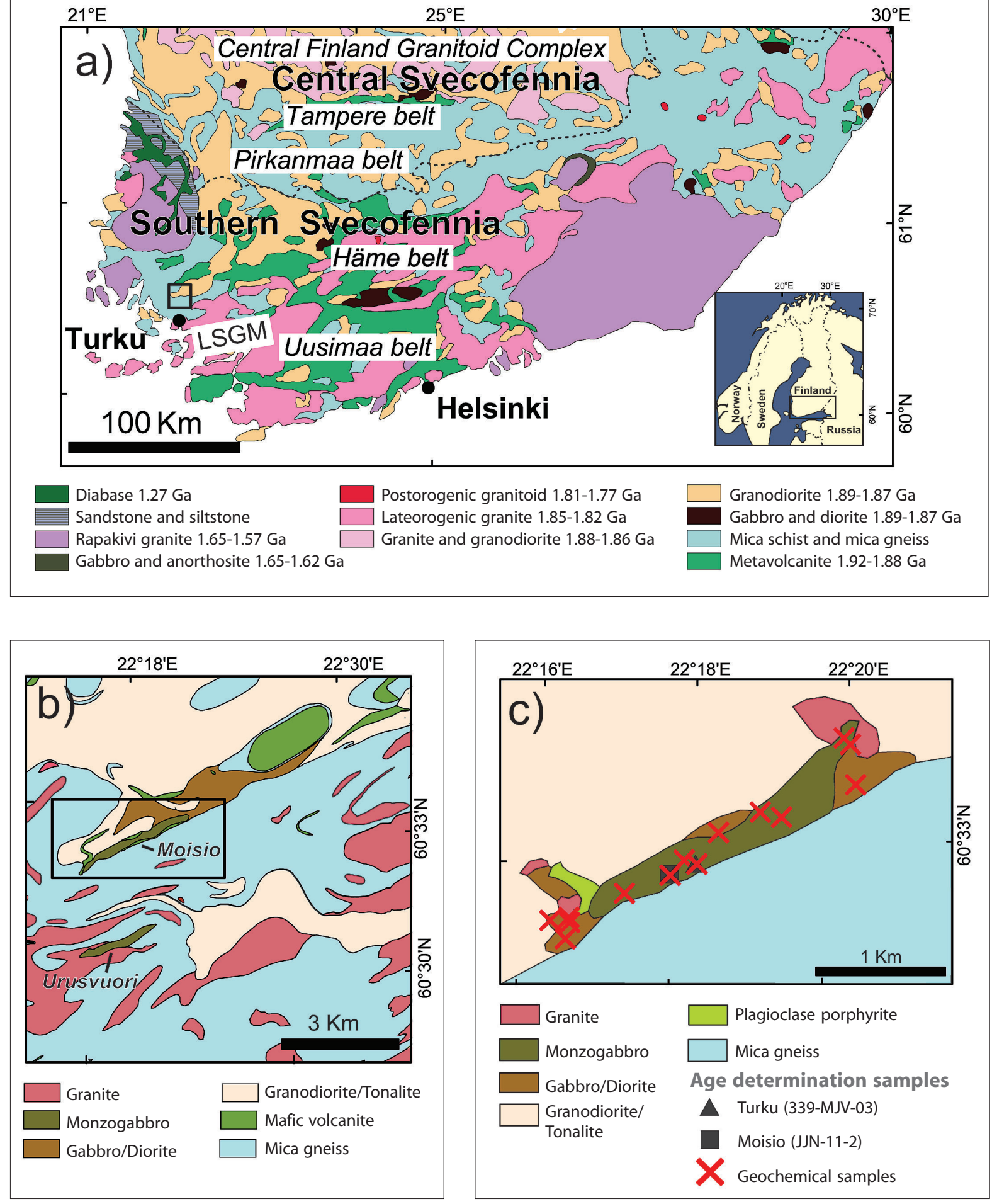

Fig. 1. a) The general geological map of southern Finland with the central and southern Svecofennia boundary indicated by broken line. The study area is shown by a rectangle. LSGM=Late Svecofennian granite-migmatite zone. b) Location of the Moisio intrusion and the postorogenic Urusvuori intrusion on the geological map. c) Detailed geological map of the Moisio study area with age determination and geochemical sample sites indicated. Figures are modified after Bedrock of Finland - DigiKP. 
plutonic rocks in SW Finland are. Provided that the volcanic rocks are of the same age, this indicates that not all the intra-orogenic magmatism comes from similar sources. The coeval felsic magmatism is derived from anatectic melting of the Svecofennian sedimentary and igneous sources (Kurhila et al., 2010).

The 1.84-1.80 Ga Svecobaltic collision led to basin inversion and crustal thickening (Lahtinen et al., 2005; Nironen \& Mänttäri, 2012). In southern Svecofennia the Svecobaltic orogeny is coeval with high-grade metamorphism and crustal melting, with peak conditions of c. $800{ }^{\circ} \mathrm{C}$ and 6 kbar at $1.83-$ 1.81 Ga (e.g. Väisänen \& Hölttä, 1999; Mouri et al., 2005; Skyttä \& Mänttäri, 2008). Therefore, granites and migmatites characterise the geology of southern Finland and has therefore been called the late-Svecofennian granite-migmatite zone (LSGM; Ehlers et al., 1993; Fig. 1a;). The $1838 \pm 4 \mathrm{Ma}$ gabbro (Pajunen et al., 2008) is ambiguously placed here in this group. Similar conditions prevailed in south-central Sweden (Högdahl et al., 2008).

The postorogenic 1.81-1.79 Ga magmatism in southern Svecofennia is characterised by LREE, Ba, $\mathrm{Sr}, \mathrm{P}$ and $\mathrm{F}$-enriched granites, monzonites, monzogabbros and lamprophyres (Eklund et al., 1998; Väisänen et al., 2000; Andersson et al., 2006a; Rutanen et al., 2011). Additionally, the $1796 \pm 9$ Ma carbonatite dykes in the Naantali area are derived from a carbonate metasomatised mantle during the postorogenic stage (Woodard, 2014).

\subsection{Geology of the study area and studied rocks}

The studied rocks are located in the western part of southern Svecofennia (Fig. 1b). The oldest rock units are metapelites and-psammites interpreted as turbidites deposited on the sea floor (Väisänen and Hölttä, 1999). These are garnet and cordierite bearing migmatites with occasional sillimanite (van Duin, 1992). The sedimentary rocks were intruded by synorogenic granitoid intrusions at c. $1.87 \mathrm{Ga}$ (Väisänen et al., 2002). The subsequent collisional event between 1.84 and $1.80 \mathrm{Ga}$ (Lahtinen et al.,
2005) formed the predominant $\sim$ E-W trending structures in the area (Väisänen \& Hölttä, 1999). The low P- high T- metamorphism culminated at $1824 \pm 5 \mathrm{Ma}$ in the Turku area (Väisänen et al., 2002) and large amounts of crustal melts were produced (e.g. Väisänen \& Hölttä, 1999; Johannes et al., 2003). Additionally, there are postorogenic intrusions, around $1.8 \mathrm{Ga}$, which are high $\mathrm{Ba}$ and Sr granites as well as mafic intrusions enriched in $\mathrm{K}$, LREE, F and P (Eklund et al., 1998); such as the $1815 \pm 2 \mathrm{Ma}$ Urusvuori monzodiorite (Väisänen et al., 2000), located just a few kilometres south of the study area. In this study a monzogabbro, gabbro and a plagioclase porphyrite from Moisio were investigated. A more detailed study area description can be found in Nevalainen (2014).

The monzogabbro (geochemical classification) outcrops in a narrow elongate NE-SW oriented $5 \mathrm{x}$ $1.5 \mathrm{~km}$ area. The granite occurs on the same outcrops (Fig. 2a). The monzogabbro contains finegrained mafic inclusions (Fig. 2b). Major minerals in the monzogabbro are hornblende, biotite, plagioclase and apatite, as well as accessory minerals titanite, magnetite, and zircon. The granite consists of K-feldspar, plagioclase, biotite and accessory minerals quartz, apatite, magnetite.

The gabbro outcrops are found at the western and eastern margins of the monzogabbro. With its variable grain size it is fairly similar to the monzogabbro in appearance and mineralogical composition with major minerals biotite, hornblende, plagioclase and locally quartz. The accessory minerals are apatite, magnetite, zircon and titanite. However, the abundances of apatite and titanite are much smaller than in the monzogabbro.

The plagioclase porphyrite is located on the western edge of the monzogabbro. It has very distinct plagioclase phenocrysts, approximately 1$2 \mathrm{~cm}$ long. Under microscope the plagioclase phenocrysts consist of plagioclase mosaic, which indicates recrystallisation during metamorphism. The matrix consists of hornblende, biotite, plagioclase and accessory minerals magnetite, zircon and apatite. 

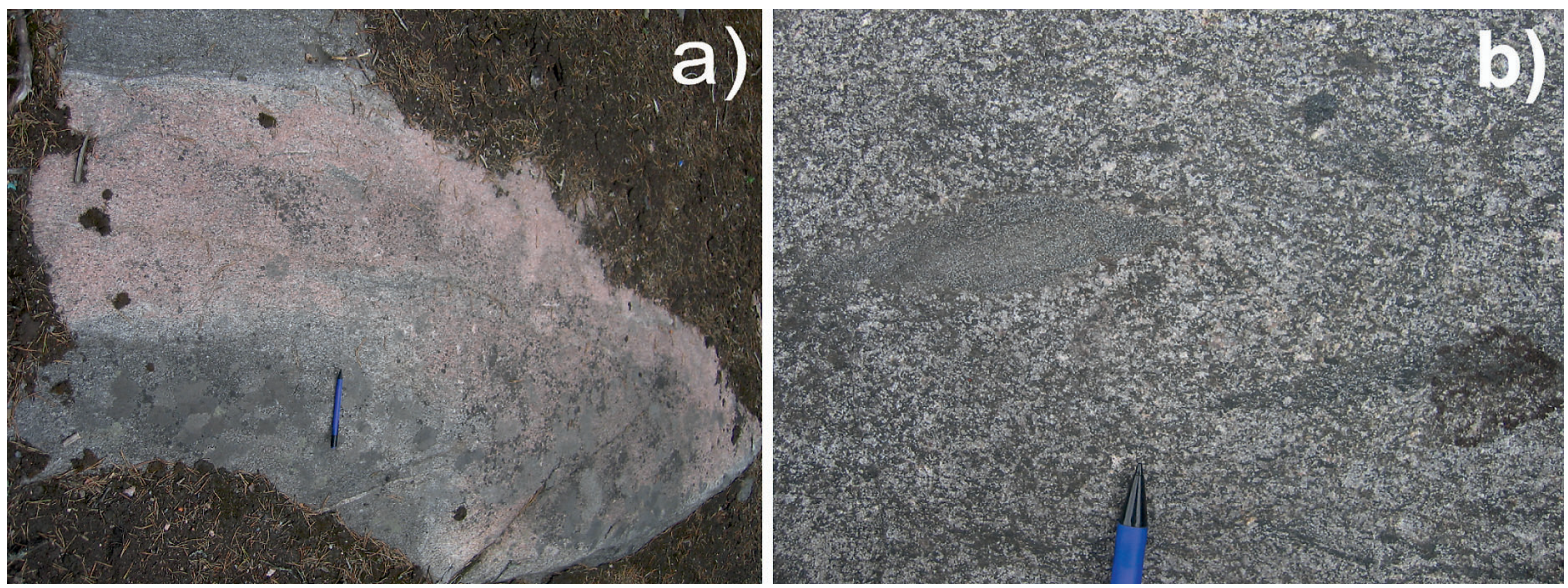

Fig. 2. Outcrop photographs of the Moisio monzogabbro, a pencil for scale: a) outcrop with mingling of monzogabbro and granite, b) fine-grained mafic inclusions and hornblende clusters.

\section{Analytical methods}

The age determinations were performed in the Finnish Isotope Geosciences Laboratory at the Geological Survey of Finland in Espoo. The instrument for the $\mathrm{U}-\mathrm{Pb}$ zircon isotope analyses was LA-MC-ICP-MS (laser ablation multi-collector inductively coupled plasma mass spectrometer). Laser ablation was performed with a New Wave UP193 Nd: YAG (neodymium-doped yttrium aluminium garnet) microprobe laser. Zircons mounted on a polished epoxy puck were ablated under a He atmosphere (gas flow $=0.2-0.31 / \mathrm{min}$ ) in a teardrop-shaped $\left(<2.5 \mathrm{~cm}^{3}\right)$ ablation cell (Horstwood et al., 2003). The He flow was lead into a Teflon mixing chamber and mixed with the Ar carrier gas (gas flow $=1.21 / \mathrm{min}$ ) and subsequently entered into the plasma torch. Ablation was done with a $20 \mu \mathrm{m}$ diameter beam with $10 \mathrm{~Hz}$ pulse frequency, and a $1.4 \mathrm{~J} / \mathrm{cm}^{2}$ energy density. Each analysis started with 30 seconds of on-mass background measurement before 60 seconds of stationary beam ablation. Secondary electron multiplier detectors analysed the ${ }^{204} \mathrm{~Pb},{ }^{206} \mathrm{~Pb},{ }^{207} \mathrm{~Pb}$ masses and a Faraday cup the ${ }^{238} \mathrm{U}$ mass. The ${ }^{235} \mathrm{U}$ mass was calculated using the ratio ${ }^{238} \mathrm{U} /{ }^{235} \mathrm{U}=$ 137.88. Isotope mass 204 was used to monitor the common ${ }^{204} \mathrm{~Pb}$. The on-mass background measurement enabled the correction for interference from ${ }^{204} \mathrm{Hg}$ in the carrier gas. Prior to the analysis session one calibration standard and one in-house calibration standard were run. This was repeated after every ten analysis sets. Calibration standards were: GJ-1 (609 \pm 1 Ma; Belousova et al., 2006) and the in-house sample was A382 (1877 $\pm 2 \mathrm{Ma}$ Patchett and Kouvo, 1986). Data reduction and calculations were done with Microsoft Excel/VBA spreadsheet routines developed by T. Andersen (Rosa et al., 2009). A more detailed method description can be found in Huhma et al. (2012).

The separated zircons were placed on an epoxy mount and polished to reveal the inner parts of the grains. The zircons were then BSE (back-scattered electrons) -imaged with the JEOL JXA-8600 SEM Superprobe at Top Analytica Ltd., Turku. The BSE images were used to study the internal structures of the zircons and to determine the LA-MC-ICP-MS analysis spots. Isoplot Ex/4.15 software (Ludwig, 2003) was used for calculating and plotting the U$\mathrm{Pb}$ isotope data. The analysed data are shown in Table 1.

The geochemical analyses were carried out by Acme Analytical Laboratories Ltd. (Acme) in Vancouver, Canada. After a lithium borate fusion and dissolution in dilute acid the major elements and chromium were analysed by ICP-ES and the other elements were analysed by ICP-MS. Additionally, fluorine was analysed by single element assay. The analytical precision is $1-5 \%$ for the major oxides and $\pm 10 \%$ for the other elements. One sample was analysed at the Geological Survey of Finland (see Table 2). 
Table 1. LA-MC-ICP-MS data for the Moisio (JJN-11-2) and Turku (339-MJV-03) samples.

Sample Moisio (JJN-11-2; N: 6722962, E: 3242143*)

$\begin{array}{lcc}\text { Analysis ID } & \mathrm{U} \text { (ppm) } & { }^{206} \mathrm{~Pb} \\ \text { Moisio 14 } & 200 & 94.1 \\ \text { Moisio 16 } & 704 & 343.7 \\ \text { Moisio 18a } & 392 & 188.6 \\ \text { Moisio 18b } & 452 & 211.2 \\ \text { Moisio 20 } & 214 & 92.8 \\ \text { Moisio 23 } & 1302 & 645.9 \\ \text { Moisio 24 } & 513 & 244.6 \\ \text { Moisio 25 } & 145 & 70.7 \\ \text { Moisio 29 } & 748 & 346.6 \\ \text { Moisio 30 } & 678 & 331 \\ \text { Moisio 30b } & 621 & 289.2 \\ \text { Moisio 32 } & 571 & 268.1 \\ \text { Moisio 32b } & 640 & 303 \\ \text { Moisio 33 } & 163 & 72.5 \\ \text { Moisio 35 } & 1026 & 496.6 \\ \text { Moisio 36 } & 1221 & 601.1 \\ \text { Moisio 39 } & 884 & 384.3 \\ \text { Moisio 46 } & 283 & 128 \\ \text { Moisio 49 } & 549 & 256.7 \\ \text { Moisio 51 } & 334 & 137.3 \\ \text { Moisio 51b } & 861 & 379.9 \\ \text { Moisio 51c } & 935 & 435.5\end{array}$

Measured ratios

$\begin{array}{cccccc}{ }^{206} \mathrm{~Pb}_{\mathrm{c}}(\%)^{\mathrm{a}} & { }^{206} \mathrm{~Pb} /{ }^{204} \mathrm{~Pb} & { }^{207} \mathrm{~Pb} /{ }^{206} \mathrm{~Pb} & 1 \sigma^{\mathrm{b}} & { }^{207} \mathrm{~Pb} /{ }^{235} \mathrm{U} & 1 \sigma^{\mathrm{b}} \\ 0.14 & 12855 & 0.11434 & 0.00068 & 5.54751 & 0.41212 \\ 0.03 & 33892 & 0.11297 & 0.00066 & 5.68885 & 0.42613 \\ 0.0018 & 35078 & 0.11281 & 0.00067 & 5.64369 & 0.42203 \\ 0.52 & 3214 & 0.11494 & 0.00081 & 5.57352 & 0.41139 \\ 0.048 & 15342 & 0.11409 & 0.00069 & 5.17495 & 0.3736 \\ 0 & 157909 & 0.11338 & 0.00066 & 5.88122 & 0.44229 \\ 0.0061 & 32146 & 0.11369 & 0.00067 & 5.67818 & 0.42112 \\ 0 & 15727 & 0.11435 & 0.00072 & 5.81751 & 0.43441 \\ 0.096 & 21991 & 0.11338 & 0.00068 & 5.56253 & 0.40998 \\ 0.026 & 136060 & 0.11353 & 0.00068 & 5.864 & 0.43878 \\ 0.3 & 4743 & 0.11397 & 0.00069 & 5.63596 & 0.41738 \\ 0.011 & 58957 & 0.11328 & 0.00055 & 5.74285 & 0.38927 \\ 0.026 & 45998 & 0.11334 & 0.00068 & 5.70706 & 0.42102 \\ 0.094 & 10953 & 0.11356 & 0.00073 & 5.41453 & 0.40705 \\ 0.00071 & 151462 & 0.11396 & 0.00067 & 5.84869 & 0.41754 \\ 0.16 & 62680 & 0.11409 & 0.00079 & 5.96773 & 0.42926 \\ 0.04 & 50181 & 0.11367 & 0.00059 & 5.22721 & 0.37699 \\ 0 & 25152 & 0.1139 & 0.00072 & 5.58721 & 0.40775 \\ 0 & 46352 & 0.11393 & 0.00072 & 5.82312 & 0.43042 \\ 0.7 & 24240 & 0.11606 & 0.00119 & 4.95201 & 0.36144 \\ 0.043 & 46836 & 0.11384 & 0.00073 & 5.37304 & 0.39159 \\ 0.0052 & 74300 & 0.1137 & 0.00072 & 5.77673 & 0.4305\end{array}$

Sample Turku (339-MJV-03; N: 6723170, E: 32442520*)

\begin{tabular}{|c|c|c|c|c|c|c|c|c|}
\hline Analysis ID & $U(p p m)$ & ${ }^{206} \mathrm{~Pb}$ & ${ }^{206} \mathrm{~Pb}_{c}(\%)^{a}$ & ${ }^{206} \mathrm{~Pb} /{ }^{204} \mathrm{~Pb}$ & ${ }^{207} \mathrm{~Pb} /{ }^{206} \mathrm{~Pb}$ & $1 \sigma^{b}$ & ${ }^{207} \mathrm{~Pb} /{ }^{235} \mathrm{U}$ & $1 \sigma^{b}$ \\
\hline Turku-8 & 93 & 30.3 & 0 & 13590 & 0.11664 & 0.00163 & 5.35979 & 0.30376 \\
\hline Turku-8b & 52 & 16.9 & 0 & 5806 & 0.11494 & 0.0016 & 5.2866 & 0.30048 \\
\hline Turku-10a & 861 & 295.1 & 0.0098 & 83727 & 0.11451 & 0.00157 & 5.49122 & 0.31886 \\
\hline Turku-10b & 83 & 26.3 & 0.0220 & 8842 & 0.11247 & 0.00155 & 5.02647 & 0.28107 \\
\hline Turku-10C & 137 & 51.5 & 0.43 & 1975 & 0.11139 & 0.00081 & 4.6518 & 0.19674 \\
\hline Turku-13 & 849 & 300.4 & 0.0150 & 85209 & 0.11457 & 0.00158 & 5.66049 & 0.33234 \\
\hline Turku-13b & 127 & 47.3 & 2.1 & 915 & 0.10419 & 0.00177 & 4.34086 & 0.1782 \\
\hline Turku-1 4 & 105 & 35.1 & 0 & 12390 & 0.11587 & 0.00162 & 5.42909 & 0.31001 \\
\hline Turku-14b & 74 & 24.6 & 0 & 9788 & 0.11615 & 0.00162 & 5.42944 & 0.31083 \\
\hline Turku-15 & 756 & 267.7 & 0 & 85593 & 0.11582 & 0.00169 & 5.40311 & 0.36057 \\
\hline Turku-15b & 621 & 258.8 & 0.1 & 14656 & 0.11216 & 0.00063 & 5.23587 & 0.18406 \\
\hline Turku-17a & 528 & 222.9 & 0.11 & 8751 & 0.11205 & 0.0006 & 5.25109 & 0.15251 \\
\hline Turku-17b & 69 & 29.7 & 0.84 & 1095 & 0.10677 & 0.00066 & 4.95643 & 0.16358 \\
\hline Turku-17c & 106 & 42.5 & 0 & 1863 & 0.11369 & 0.00069 & 5.09077 & 0.14478 \\
\hline Turku-20 & 578 & 202.5 & 0.0200 & 55398 & 0.11575 & 0.00169 & 5.33374 & 0.35259 \\
\hline Turku-20b & 96 & 28.9 & 0.9900 & 26370 & 0,11313 & 0.00196 & 4.7278 & 0.26306 \\
\hline Turku-22a & 525 & 182.5 & 0 & 272753 & 0.1156 & 0.00168 & 5.27667 & 0.34673 \\
\hline Turku-22b & 378 & 132.9 & 0.0220 & 66327 & 0.11566 & 0.00169 & 5.34561 & 0.35372 \\
\hline Turku-23 & 444 & 155.2 & 0.0340 & 395404 & 0.11566 & 0.00169 & 5.30988 & 0.35018 \\
\hline Turku-25a & 550 & 190.9 & 0.0160 & 100588 & 0.11456 & 0.00166 & 5.23262 & 0.34225 \\
\hline Turku-25b & 1514 & 11.4 & 0 & 5456 & 0.12401 & 0.01597 & 0.13063 & 0.13161 \\
\hline Turku-26 & 1286 & 451.9 & 0.0150 & 120312 & 0.1157 & 0.00168 & 5.34319 & 0.35273 \\
\hline Turku-28a & 574 & 203.7 & 0.0071 & 63371 & 0.11537 & 0.00168 & 5.38657 & 0.35763 \\
\hline Turku-28b & 748 & 265.4 & 0.0250 & 77442 & 0.11574 & 0.00169 & 5.38427 & 0.35666 \\
\hline Turku-29a & 495 & 174.4 & 0.0013 & 67062 & 0.11544 & 0.00168 & 5.34002 & 0.35212 \\
\hline Turku-29b & 90 & 30.1 & 0.0990 & 8552 & 0.11616 & 0.0017 & 5.12597 & 0.33008 \\
\hline Turku-29c & 511 & 179.2 & 0 & 52420 & 0.11534 & 0.00168 & 5.33986 & 0.35336 \\
\hline Turku-30 & 36 & 12.5 & 8.9000 & 608 & 0.10174 & 0.00435 & 4.51139 & 0.31752 \\
\hline
\end{tabular}

Note: Analyses in red were ommitted from concordia age calculations. Analyses in green and purple were obtained from the zircon rims.

a Precentage of common ${ }^{206} \mathrm{~Pb}$ in measured ${ }^{206} \mathrm{~Pb}$ calculated from the ${ }^{204} \mathrm{~Pb}$ signal using age-related common lead after model by Stacey \& Kramers (1975). b Errors are absolute $1 \sigma$ values.

c Error correlation for $207 \mathrm{~Pb} / 235 \mathrm{U}$ versus $206 \mathrm{~Pb} / 238 \mathrm{U}$ ratios.

$\mathrm{d}$ The precentage of discordance from the concordia, relative to the centroid of the ellipse.

* Coordinates in Finnish zone 3 (KKJ 3) 
Discordance Ages (Ma)

\begin{tabular}{|c|c|c|c|c|c|c|c|c|c|}
\hline${ }^{206} \mathrm{~Pb} /{ }^{238} \mathrm{U}$ & $1 \sigma^{b}$ & $\rho^{c}$ & Central $(\%)^{d}$ & ${ }^{207} \mathrm{~Pb} /{ }^{206} \mathrm{~Pb}$ & $1 \sigma^{b}$ & ${ }^{207} \mathrm{~Pb} /{ }^{235} \mathrm{U}$ & $1 \sigma^{b}$ & $206 \mathrm{~Pb} / 238 \mathrm{U}$ & $1 \sigma$ \\
\hline 0.36522 & 0.02727 & 0.997 & 10 & 1848 & 10 & 1930 & 65 & 2007 & 129 \\
\hline 0.36283 & 0.02705 & 0.997 & 9.5 & 1845 & 10 & 1923 & 65 & 1996 & 128 \\
\hline 0.32897 & 0.02367 & 0.997 & -2 & 1866 & 11 & 1849 & 61 & 1833 & 115 \\
\hline 0.37621 & 0.02821 & 0.997 & 12.9 & 1854 & 10 & 1958 & 65 & 2059 & 132 \\
\hline 0.36224 & 0.02678 & 0.997 & 8.4 & 1859 & 11 & 1928 & 64 & 1993 & 127 \\
\hline 0.3746 & 0.02794 & 0.997 & 12.2 & 1857 & 11 & 1956 & 65 & 2051 & 131 \\
\hline 0.35864 & 0.02647 & 0.997 & 7 & 1864 & 10 & 1922 & 64 & 1976 & 126 \\
\hline 0.36768 & 0.02486 & 0.997 & 10.4 & 1853 & 8 & 1938 & 59 & 2018 & 117 \\
\hline 0.36519 & 0.02685 & 0.997 & 9.6 & 1854 & 10 & 1932 & 64 & 2007 & 127 \\
\hline 0.3458 & 0.0259 & 0.996 & 3.6 & 1857 & 11 & 1887 & 64 & 1915 & 124 \\
\hline 0.37224 & 0.02648 & 0.997 & 11.1 & 1863 & 10 & 1954 & 62 & 2040 & 124 \\
\hline 0.30945 & 0.02236 & 0.99 & -9.5 & 1896 & 18 & 1811 & 62 & 1738 & 110 \\
\hline 0.34232 & 0.02485 & 0.996 & 2.2 & 1862 & 11 & 1881 & 62 & 1898 & 119 \\
\hline 0.36848 & 0.02736 & 0.996 & 10.2 & 1859 & 11 & 1943 & 65 & 2022 & 12 \\
\hline
\end{tabular}

\begin{tabular}{|c|c|c|c|c|c|c|c|c|c|}
\hline${ }^{206} \mathrm{~Pb} /{ }^{238} \mathrm{U}$ & $1 \sigma^{b}$ & $\rho^{c}$ & Central $(\%)^{d}$ & ${ }^{207} \mathrm{~Pb} /{ }^{206} \mathrm{~Pb}$ & $1 \sigma^{b}$ & ${ }^{207} \mathrm{~Pb} / 235 \mathrm{U}$ & $1 \sigma^{b}$ & ${ }^{206} \mathrm{~Pb} /{ }^{238} \mathrm{U}$ & $1 \sigma^{b}$ \\
\hline 0.33327 & 0.01831 & 0.969 & -3.1 & 1905 & 24 & 1878 & 48 & 1854 & 89 \\
\hline 0.33359 & 0.01838 & 0.970 & -1.4 & 1879 & 24 & 1867 & 49 & 1856 & 89 \\
\hline 0.34779 & 0.01962 & 0.972 & 3.2 & 1872 & 24 & 1899 & 50 & 1924 & 94 \\
\hline 0.30289 & 0.01262 & 0.985 & -7.3 & 1822 & 13 & 1759 & 35 & 1706 & 62 \\
\hline 0.35834 & 0.02045 & 0.972 & 6.3 & 1873 & 24 & 1925 & 51 & 1974 & 97 \\
\hline 0.30218 & 0.01129 & 0.911 & 0.1 & 1700 & 31 & 1701 & 34 & 1702 & 56 \\
\hline 0.33834 & 0.02203 & 0.976 & -0.9 & 1893 & 26 & 1885 & 57 & 1879 & 106 \\
\hline 0.33857 & 0.01175 & 0.987 & 2.8 & 1835 & 10 & 1858 & 30 & 1880 & 57 \\
\hline 0.33989 & 0.0097 & 0.983 & 3.4 & 1833 & 9 & 1861 & 25 & 1886 & 47 \\
\hline 0.33669 & 0.01092 & 0.983 & 8.3 & 1745 & 11 & 1812 & 28 & 1871 & 53 \\
\hline 0.32475 & 0.00903 & 0.977 & -2.9 & 1859 & 10 & 1835 & 24 & 1813 & 44 \\
\hline 0.3342 & 0.02155 & 0.975 & -2 & 1892 & 25 & 1874 & 57 & 1859 & 104 \\
\hline 0.00764 & 0.00763 & 0.992 & -97.9 & 2015 & 235 & 125 & 118 & 49 & 49 \\
\hline 0.33495 & 0.02157 & 0.975 & -1.7 & 1891 & 26 & 1876 & 56 & 1862 & 104 \\
\hline 0.33863 & 0.02194 & 0.976 & -0.3 & 1886 & 25 & 1883 & 57 & 1880 & 106 \\
\hline 0.33739 & 0.0218 & 0.976 & -1.1 & 1891 & 25 & 1882 & 57 & 1874 & 105 \\
\hline 0.33548 & 0.02158 & 0.975 & -1.3 & 1887 & 25 & 1875 & 56 & 1865 & 104 \\
\hline 0.32005 & 0.02007 & 0.974 & -6.5 & 1898 & 25 & 1840 & 55 & 1790 & 98 \\
\hline 0.33577 & 0.02168 & 0.976 & -1.2 & 1885 & 25 & 1875 & 57 & 1866 & 105 \\
\hline 0.3216 & 0.01798 & 0.794 & 9.8 & 1656 & 75 & 1733 & 58 & 1798 & 88 \\
\hline
\end{tabular}


Table 2. Geochemical analyses from the Moisio area.

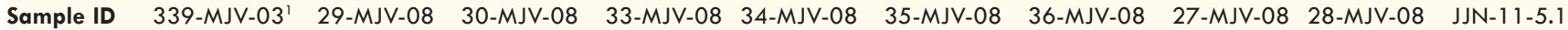

\begin{tabular}{|c|c|c|c|c|c|c|c|c|c|c|}
\hline Rock type & $\mathrm{MzDr}$ & $\mathrm{MzGb}$ & $M z G b$ & $\mathrm{MzGb}$ & $\mathrm{Dr}$ & $\mathrm{MzGb}$ & $\mathrm{MzGb}$ & PI-por & $\mathrm{Gb}$ & $\mathrm{MzGb}$ \\
\hline Locality & Moisio & Moisio & Moisio & Moisio & Moisio & Moisio & Moisio & Moisio & Moisio & Moisio \\
\hline N-Coord* & 6723170 & 6722754 & 6722951 & 6723128 & 6723456 & 6723711 & 6723651 & 6722517 & 6722455 & 6724601 \\
\hline E-Coord* & 3242520 & 3241695 & 3242155 & 3242377 & 3242783 & 3243320 & 3243577 & 3241095 & 3241104 & 3244362 \\
\hline $\mathrm{SiO}_{2}(w t \%)$ & 49 & 45.3 & 48.67 & 47.35 & 49.89 & 48.56 & 38.02 & 46.69 & 50.23 & 50.47 \\
\hline $\mathrm{Al}_{2} \mathrm{O}_{3}(\mathrm{wt} \%)$ & 17.2 & 15.73 & 17.11 & 18.13 & 13.81 & 17.24 & 14.7 & 19.5 & 17.79 & 19.5 \\
\hline $\mathrm{Fe}_{2} \mathrm{O}_{3}(w t \%)$ & 10.14 & 13.22 & 11.34 & 12.23 & 11.18 & 11.31 & 16.56 & 11.62 & 9.84 & 9.14 \\
\hline $\mathrm{MgO}(\mathrm{wt} \%)$ & 4.56 & 7 & 4.27 & 4.79 & 8.96 & 4.57 & 8 & 4.31 & 5.88 & 3.9 \\
\hline $\mathrm{CaO}(w t \%)$ & 7.45 & 8.59 & 7 & 8.33 & 9.68 & 7.92 & 11.81 & 9.39 & 8.62 & 6.93 \\
\hline $\mathrm{Na}_{2} \mathrm{O}(w t \%)$ & 4.56 & 2.71 & 4,44 & 3.94 & 3.12 & 3.99 & 2.26 & 3.3 & 3.44 & 4.35 \\
\hline $\mathrm{K}_{2} \mathrm{O}(\mathrm{wt} \%)$ & 2.02 & 2.46 & 2.27 & 1.65 & 0.92 & 1.75 & 1.31 & 2.07 & 1.62 & 2.04 \\
\hline $\mathrm{TiO}_{2}(\mathrm{wt} \%)$ & 1.86 & 2.07 & 2.11 & 1.84 & 1.03 & 2.49 & 3.17 & 1.47 & 1.18 & 1.76 \\
\hline$P_{2} O_{5}(w t \%)$ & 1.36 & 1.26 & 1.61 & 0.91 & 0.21 & 1.04 & 2.74 & 0.33 & 0.21 & 0.78 \\
\hline $\mathrm{MnO}(\mathrm{wt} \%)$ & 0.15 & 0.19 & 0.16 & 0.14 & 0.18 & 0.14 & 0.15 & 0.1 & 0.15 & 0.13 \\
\hline Sc (ppm) & 12.2 & 27 & 14 & 22 & 33 & 26 & 34 & 25 & 22 & 9 \\
\hline $\mathrm{Ba}(\mathrm{ppm})$ & 1348 & 345 & 866 & 711 & 253 & 672 & 387 & 315 & 279 & 1318 \\
\hline Cs (ppm) & n.a. & 4.9 & 6 & 0.6 & 1.1 & 0.9 & 2.3 & 11.4 & 5.3 & 2.9 \\
\hline Hf (ppm) & 3.98 & 2.5 & 6.6 & 3 & 2.6 & 2.7 & 2.4 & 2.8 & 2 & 0.8 \\
\hline Nb (ppm) & 22.4 & 11.5 & 33 & 18 & 5.6 & 21.9 & 11 & 7.9 & 8.9 & 13.6 \\
\hline $\mathbf{R b}$ (ppm) & 65.9 & 97.6 & 82.7 & 34.7 & 19.3 & 47.9 & 30.2 & 94.7 & 58.2 & 85.7 \\
\hline Sr (ppm) & 2464 & 758.1 & 1490.2 & 1030.2 & 437.5 & 1168.5 & 1398.4 & 521.9 & 840.9 & 2182.7 \\
\hline Ta (ppm) & 0.84 & 0.4 & 1.3 & 0.9 & 0.3 & 1.1 & 0.4 & 0.4 & 0.4 & 0.6 \\
\hline Th (ppm) & 4.16 & 2.1 & 7.1 & 1.8 & 2.1 & 1.2 & 1.3 & 1.3 & 1.8 & 1.2 \\
\hline $\mathbf{U}(\mathbf{p p m})$ & 1.17 & 1.6 & 2.8 & 0.8 & 0.9 & 0.8 & 0.7 & 0.6 & 1.2 & 1 \\
\hline$V(p p m)$ & 212 & 296 & 173 & 220 & 255 & 192 & 410 & 254 & 226 & 107 \\
\hline $\mathrm{Zr}$ (ppm) & 192 & 102.1 & 345.8 & 121.8 & 92.1 & 124.8 & 75.5 & 102.4 & 97.2 & 32.8 \\
\hline$Y(p p m)$ & 29.5 & 23.2 & 29.7 & 28.6 & 15.3 & 29 & 31.2 & 19.7 & 14.8 & 19.8 \\
\hline La (ppm) & 123 & 34.3 & 98.7 & 49.9 & 16.3 & 61.5 & 49.5 & 14.5 & 21.1 & 59.5 \\
\hline $\mathrm{Ce}$ (ppm) & 278 & 88.9 & 227 & 129.3 & 36.6 & 148.1 & 133.3 & 32.1 & 53.8 & 146.8 \\
\hline $\operatorname{Pr}(p p m)$ & 33.2 & 13.15 & 28.68 & 17,25 & 4.9 & 20.43 & 20.98 & 4.4 & 7.58 & 17.94 \\
\hline Nd (ppm) & 129 & 60.6 & 116.1 & 71.3 & 18.8 & 84.4 & 100.3 & 20.8 & 33.5 & 67.9 \\
\hline Sm (ppm) & 18.3 & 10.59 & 17.07 & 12.41 & 3.97 & 14.5 & 18.84 & 4,32 & 6.08 & 10.87 \\
\hline Eu (ppm) & 4.35 & 2.92 & 4.26 & 3.22 & 1.18 & 3.64 & 5.09 & 1.39 & 1.82 & 3.45 \\
\hline Gd (ppm) & 14.6 & 9.06 & 13.34 & 9.21 & 3.4 & 10.42 & 14.04 & 4.53 & 5.02 & 7.34 \\
\hline Tb (ppm) & 1.62 & 1.07 & 1.4 & 1.23 & 0.54 & 1.32 & 1.68 & 0.67 & 0.66 & 0.98 \\
\hline Dy (ppm) & 5.93 & 5 & 6.14 & 5.97 & 2.93 & 6.17 & 7.23 & 3.54 & 3.02 & 4.35 \\
\hline Ho (ppm) & 1.06 & 0.84 & 1.01 & 1.02 & 0.55 & 1 & 1.14 & 0.69 & 0.54 & 0.78 \\
\hline Er (ppm) & 2.33 & 2.04 & 2.54 & 2.76 & 1.62 & 2.62 & 2.63 & 2.1 & 1.42 & 2.05 \\
\hline $\operatorname{Tm}$ (ppm) & 0.3 & 0,28 & 0.35 & 0.37 & 0.23 & 0.35 & 0.34 & 0.32 & 0.2 & 0.27 \\
\hline Yb (ppm) & 1.94 & 1.65 & 2.17 & 2.15 & 1.38 & 2.03 & 1.97 & 1.89 & 1.16 & 1.43 \\
\hline Lu (ppm) & 0.29 & 0.23 & 0.32 & 0.32 & 0.21 & 0.28 & 0.26 & 0.29 & 0.18 & 0.2 \\
\hline $\mathrm{Cr}(\mathrm{ppm})^{4}$ & 9 & 55 & $<13.7$ & 13.7 & 329.3 & $<13.7$ & 20.5 & 109 & 62 & 123.2 \\
\hline $\mathrm{Ni}($ ppm) & 22 & 64 & 9.1 & 25.9 & 36.3 & 12.8 & 38.4 & 25.5 & 43.6 & 38 \\
\hline$F(p p m)$ & 2490 & 2080 & 2610 & 2170 & 820 & 3530 & 7120 & 620 & 940 & 2400 \\
\hline
\end{tabular}

\footnotetext{
1 from Väisänen (2004) where the laboratory and analytical methods can be found; ${ }^{2}$ Standard with expected concentrations; ${ }^{3}$ Mean of two STD-SO 18 analyses; ${ }^{4}$ calculated from $\mathrm{Cr}_{2} \mathrm{O}_{3}$

* Coordinates in the Finnish zone 3 (KKJ 3); **Normalizing values after Boynton (1984); < = value below the detection limit; n.a. = not analyzed; $\mathrm{MzDr}=$ Monzodiorite, $\mathrm{MzGb}=$ Monzogabbro, $\mathrm{Gb}=\mathrm{Gabbro}, \mathrm{Dr}=$ Diorite, $\mathrm{Pl}$-por= Plagiolase porphyrite
} 
JJN-11-13 JJN-11-25 JJN-11-36 JJN-11-42 STD-SO182 STD-SO18

\begin{tabular}{|c|c|c|c|c|c|}
\hline $\mathrm{Gb}$ & $\mathrm{Gb}$ & $\mathrm{Gb}$ & Gb & & \\
\hline Moisio & Moisio & Moisio & Moisio & & \\
\hline 6722261 & 6722406 & 6724517 & 6724049 & & \\
\hline 3241051 & 3240773 & 3244424 & 3244462 & & \\
\hline 49.63 & 54.34 & 47.57 & 50.6 & 5847 & 58.27 \\
\hline 18.16 & 18.54 & 13.57 & 14,86 & 14.23 & 14.1 \\
\hline 11.14 & 9.71 & 12.29 & 10.67 & 7.67 & 7.65 \\
\hline 4.11 & 2.53 & 9.41 & 8.8 & 3.35 & 3.38 \\
\hline 10.02 & 7.52 & 10.15 & 7.94 & 6.42 & 6.34 \\
\hline 3.2 & 3.11 & 1.81 & 3.08 & 3.71 & 3.46 \\
\hline 1.06 & 1.53 & 1.85 & 1.77 & 2.17 & 2.15 \\
\hline 1.39 & 1.48 & 1.26 & 0.94 & 0.69 & 0.69 \\
\hline 0.31 & 0.35 & 0.31 & 0.16 & 0.83 & 0.8 \\
\hline 0.15 & 0.16 & 0.21 & 0.17 & 0.39 & 0.4 \\
\hline 22 & 19 & 37 & 28 & 25 & 24 \\
\hline 216 & 292 & 177 & 256 & 514 & 506 \\
\hline 0.3 & 8.6 & 1.8 & 3.8 & 7.1 & 6.7 \\
\hline 2.5 & 4.3 & 2.5 & 2.4 & 9.8 & 9.6 \\
\hline 6.8 & 9.7 & 7.3 & 4.5 & 21.3 & 19,1 \\
\hline 8.8 & 90.5 & 68.4 & 65 & 28.7 & 28.0 \\
\hline 661.8 & 617.3 & 283 & 655.2 & 407.4 & 435.7 \\
\hline 0.5 & 0.6 & 0.5 & 0.4 & 7.4 & 6.7 \\
\hline 1.5 & 6.4 & 4.4 & 2.8 & 9.9 & 9.8 \\
\hline 1 & 2.6 & 2.2 & 1.3 & 16.4 & 16.3 \\
\hline 208 & 178 & 292 & 177 & 200 & 189 \\
\hline 99.9 & 155.6 & 96.9 & 95.6 & 280 & 285.7 \\
\hline 21.9 & 28.4 & 22,2 & 16.6 & 31 & 29.9 \\
\hline 17.9 & 28.7 & 17.1 & 15.4 & 12.3 & 13.2 \\
\hline 39.5 & 61.6 & 39.4 & 34.7 & 27.1 & 28.2 \\
\hline 4.84 & 7.9 & 5.08 & 4.08 & 3.45 & 3.23 \\
\hline 18.5 & 33.1 & 22.3 & 16.5 & 14 & 13.9 \\
\hline 4.71 & 6.96 & 4.72 & 3.35 & 3 & 2.84 \\
\hline 1.59 & 2.04 & 1.36 & 1.05 & 0.89 & 0.84 \\
\hline 4.7 & 6.56 & 4.46 & 3.36 & 2.93 & 2.98 \\
\hline 0.76 & 1.04 & 0.81 & 0.56 & 0.53 & 0.52 \\
\hline 3.87 & 5.46 & 4.17 & 2.82 & 3 & 2.84 \\
\hline 0.87 & 1.19 & 0.95 & 0.65 & 0.62 & 0.65 \\
\hline 2.41 & 3.36 & 2.64 & 1.94 & 1.84 & 1.82 \\
\hline 0.32 & 0.48 & 0.4 & 0.27 & 0.27 & 0.29 \\
\hline 1.94 & 2.86 & 2.23 & 1.52 & 1.79 & 1.81 \\
\hline 0.34 & 0.47 & 0.36 & 0.28 & 0.27 & 0.29 \\
\hline 109.5 & 34.2 & 595.3 & 225.8 & 3763 & 3756 \\
\hline 31 & $<20$ & 127 & 57 & 44 & 40 \\
\hline 700 & 1100 & 700 & 500 & n.a. & n.a. \\
\hline
\end{tabular}

The geochemical data were plotted using the GCDkit 3.00 software (Janoušek et al., 2006). The analysed data are shown in Table 2.

\section{Single-grain zircon $\mathrm{U}-\mathrm{Pb}$ results}

Two monzogabbro samples (Moisio, JJN-11-2 and Turku, 339-MJV-03), were collected for geochronological purposes (Fig. 1c). The sample Moisio is a monzogabbro from an outcrop which also contains granite parts. The sample Turku is a monzogabbro from an outcrop with fine-grained mafic inclusions and large quartz grain clusters.

\subsection{Moisio sample (JJN-11-2)}

The zircon grains are mainly euhedral to subrounded, prismatic and their colour varies from transparent to brown. The grains are $100-400 \mu \mathrm{m}$ in length, and rounded grains tend to be smaller. A few of the transparent grains have thin brown bands perpendicular to the c-axis. The BSE images show a growth zoning and occasional fracturing and metamict alteration (Fig. 3a-b). Core and rim parts as well as dark inclusions are also found in some of the grains but no obvious xenocrystic cores were identified. A few of the grains have lighter rims which are overprinting earlier zoning. However, these were too thin to fit a $20 \mu \mathrm{m}$ laser spot.

Altogether, 22 spots out of 17 zircon grains were analysed (Table 1). Due to anomalous ${ }^{207} \mathrm{~Pb} /{ }^{206} \mathrm{~Pb}$ ages and large $1 \sigma$ errors a few analyses were discarded. The analysis spot $18 \mathrm{~b}$ which is from a BSE-bright rim gives a ${ }^{207} \mathrm{~Pb} /{ }^{206} \mathrm{~Pb} 1 \sigma$ age of 1879 $\pm 12 \mathrm{Ma}$. The core of the same grain gives a ${ }^{207} \mathrm{~Pb} /$ ${ }^{206} \mathrm{~Pb} 1 \sigma$ age of $1848 \pm 10 \mathrm{Ma}$ (Fig. 3a). Due to high common $\mathrm{Pb}$ value and sample inhomogeneity the old rim provided an anomalous age (Table 1) and was discarded. Similarly, the ${ }^{207} \mathrm{~Pb} /{ }^{206} \mathrm{~Pb} 1 \sigma$ age for analysis 51 was $1896 \pm 18 \mathrm{Ma}$, whereas the two other analyses from the grain 51 (51b and 51c) gave $1 \sigma$ ages of $1862 \pm 11 \mathrm{Ma}$ and $1859 \pm 11 \mathrm{Ma}$ (Table 1). Moreover, the analysis spot 51 has a high common $\mathrm{Pb}$ value compared to the analysis spots $51 \mathrm{~b}$ and 51c. In conclusion, 20 analyses produced 
a concordia $2 \sigma$ age of $1859.9 \pm 5.2 \mathrm{Ma}$ (mean square weighted deviation, MSWD = 17), a TeraWasserburg $2 \sigma$ age of $1859.8 \pm 5.2 \mathrm{Ma}(\mathrm{MSWD}=$ 20; not shown) and a weighted average ${ }^{207} \mathrm{~Pb} /{ }^{206} \mathrm{~Pb}$ $95 \%$ conf. age of $1858.7 \pm 4.5$ (MSWD = 0.42; Fig. 17). As the MSWD values are fairly high for the concordia ages, a weighted average age of 1859 $\pm 5 \mathrm{Ma}$ is regarded as the best estimate for the crystallisation age (Fig 4a).

\subsection{Turku sample (339-MJV-03)}

The grain morphology of the Turku sample is quite different from the Moisio zircons. The grain size varies between 100 and $300 \mu \mathrm{m}$. The zircons are dominantly anhedral with abundant resorption features, and some grains are merely broken fragments of larger zircon grains. The zoning is not
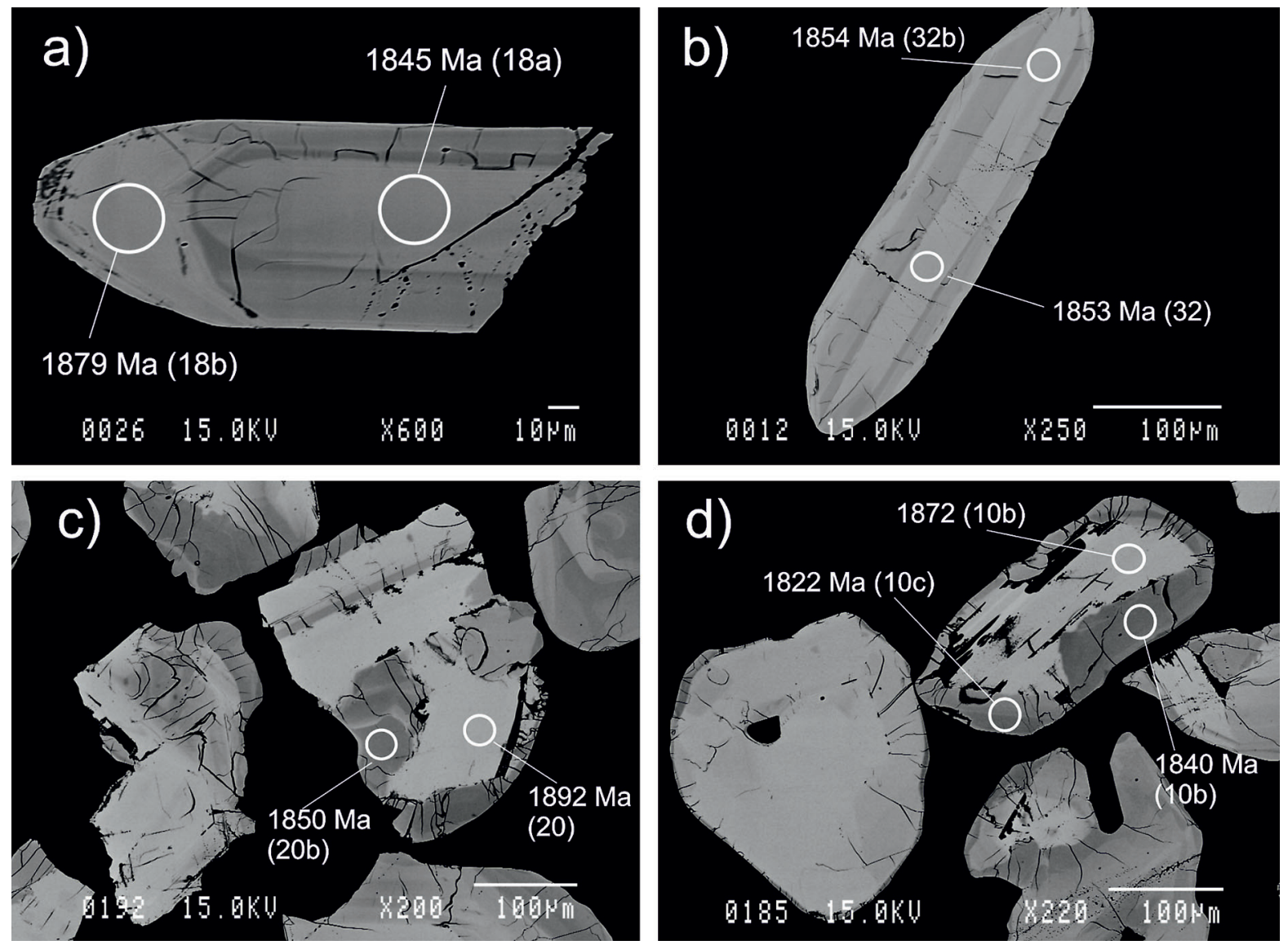

Fig. 3. BSE-images of selected zircons, a) and b) are from the Moisio sample and c) and d) are from the Turku sample. Analysis spots are circled with ${ }^{207} \mathrm{~Pb} /{ }^{206} \mathrm{~Pb}$ ages indicated, see more details in Table 1.

as clearly magmatic as in the Moisio sample grains. In contrast, the zoning is irregular, patchy and in some grains it is truncated by new growth. Metamict alteration is visible at the tips of fractures and at the boundaries of the BSE-dark and BSE-bright parts. Fracturing is common and shows preference to the darker coloured parts of the grains. There are separate core/rim parts, and often the rims are darker than the core areas (Figs 3c-d).

A total of 28 analyses were carried out from 14 zircons (Fig. 4c-e). The age determination data show four anomalously old or young $1 \sigma^{207} \mathrm{~Pb} /{ }^{206} \mathrm{~Pb}$ ages: $2015 \pm 235 \mathrm{Ma}, 1745 \pm 11 \mathrm{Ma}, 1700 \pm 31 \mathrm{Ma}$ and $1656 \pm 75$ Ma. All of these analysis show high common lead values compared to the rest of the data and were omitted from further calculations (Fig. 4b; Table 1).

The remaining 24 analyses show a large spread 

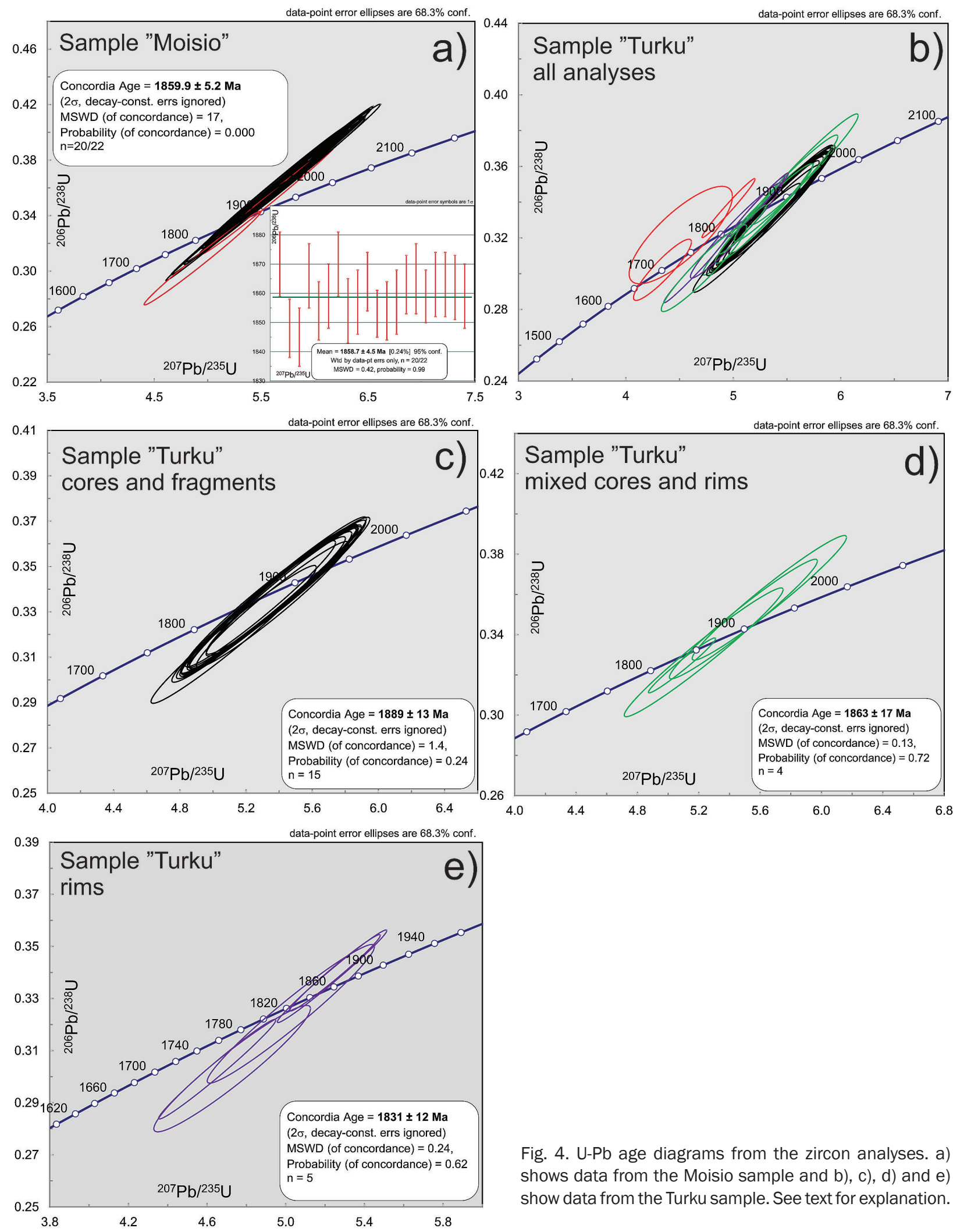

Fig. 4. U-Pb age diagrams from the zircon analyses. a) shows data from the Moisio sample and b), c), d) and e) show data from the Turku sample. See text for explanation. 
of ${ }^{207} \mathrm{~Pb} /{ }^{206} \mathrm{~Pb}$ ages between c. 1.91 and $1.82 \mathrm{Ga}$. Using 15 analyses from the core domains yielded a concordia age of $1889 \pm 13 \mathrm{Ma}(2 \sigma ; \mathrm{MSWD}=1.4$; Fig. 4c).

The nine remaining analyses appear to fall in two age groups. Four analyses, including one point from a rim, form a group with a concordia age of $1863 \pm 17$ Ma $(2 \sigma ; M S W D=0.13$; Fig. 4d). The final five rim analyses fall in a group with concordia age of $1831 \pm 12 \mathrm{Ma}(2 \sigma ; \mathrm{MSWD}=0.24$; Fig. 4e). The Tera-Wasserburg plots and weighted average ages gave similar ages with low MSWD values. Therefore, the concordia ages are preferred here.

\section{Geochemistry}

A total of 14 whole-rock geochemical analyses from the Moisio area were examined. The data were compared with the synorogenic Uusikaupunki diorite (1872 $\pm 3 \mathrm{Ma}$; Väisänen et al., 2012b), the intra-orogenic Rauma and Korpo diorites (1865 \pm $9 \mathrm{Ma}$ and $1852 \pm 4 \mathrm{Ma}$, respectively; Väisänen et al., 2012a) and the post-orogenic Urusvuori monzodiorite (1815 \pm 2 Ma; Väisänen et al., 2000).

\subsection{Major elements}

The $\mathrm{SiO}_{2}$ content of the Moisio data varies from 38 wt $\%$ to 55 wt $\%$ (Table 2). In the total alkali vs. silica diagram (TAS; Fig. 5) the data forms two groups: one group of the data plots predominantly as monzogabbro and another group plots both in the gabbro and gabbroic diorite fields. Hereafter that group is collectively called gabbro. In the $\mathrm{K}_{2} \mathrm{O}$ vs. $\mathrm{SiO}_{2}$ diagram the data show calc-alkaline to shoshonitic affinity. The monzogabbro samples show high $\mathrm{P}_{2} \mathrm{O}_{5}$ and $\mathrm{TiO}_{2}$ concentrations that is not seen in the gabbro samples (Fig. 6).

Many of the elements in the monzogabbro group plot between the synorogenic and the

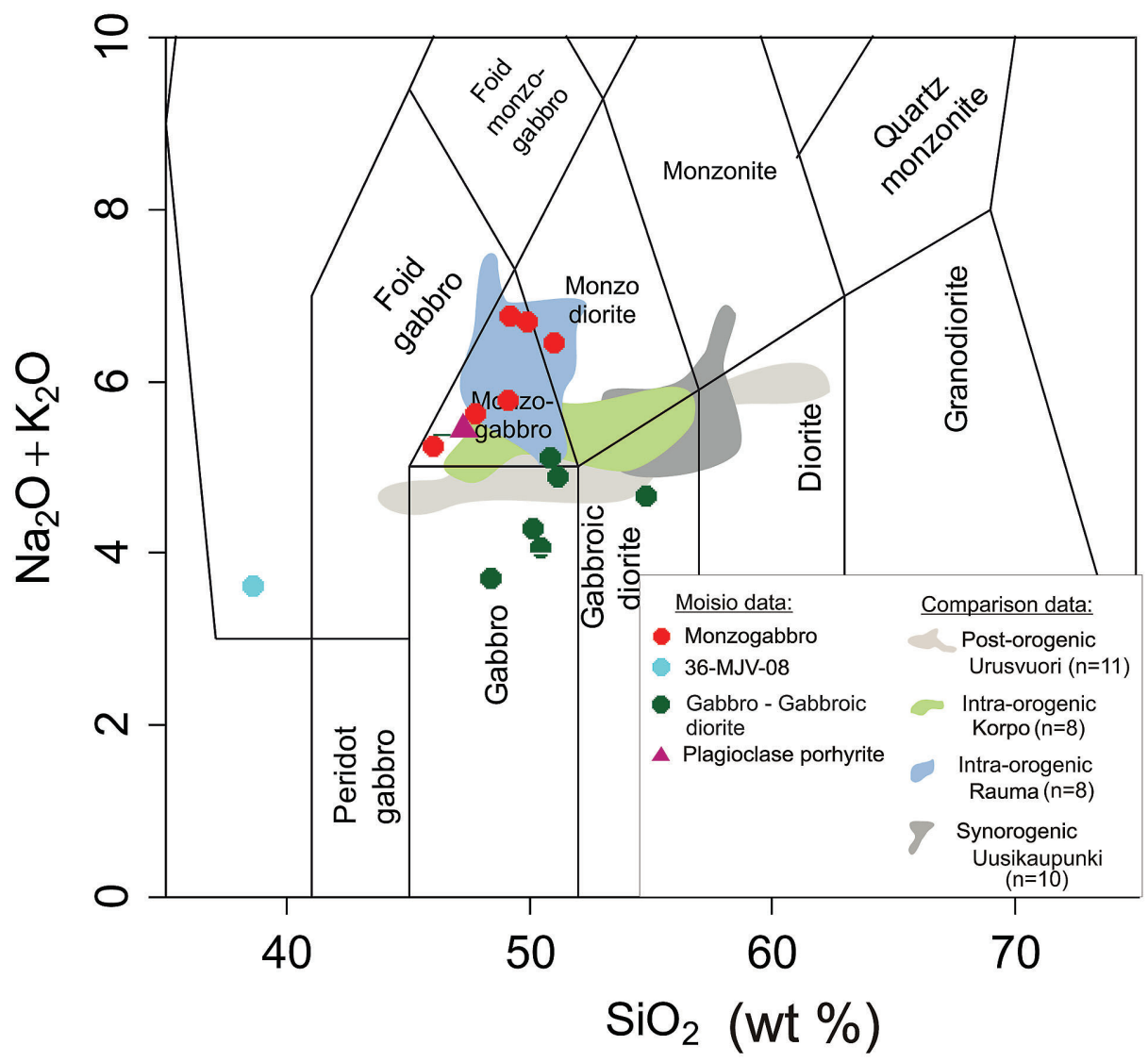

Fig. 5. The total alkalis vs silica classification diagram (TAS) of the studied rocks (after Middlemost, 1985). 

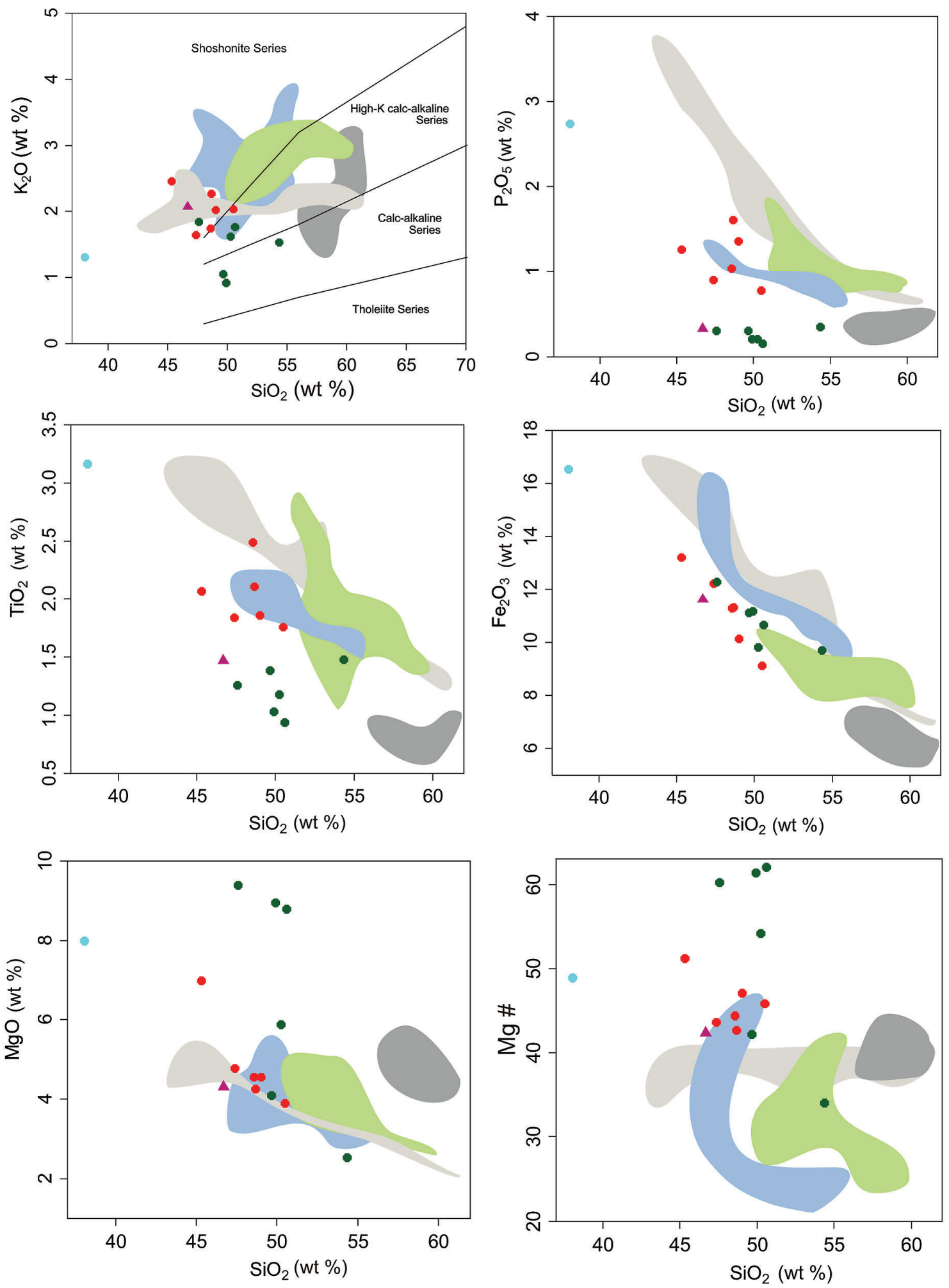

Fig. 6. Selected major element diagrams. $\mathrm{K}_{2} \mathrm{O}$ vs $\mathrm{SiO}_{2}$ classification after Peccerillo \& Taylor (1976). Mg\# calculated with GCDkit-software (Janoušek et al., 2006). Symbols and fields as in Fig. 5. 
postorogenic data. Clearly, the monzogabbro samples show relatively similar high $\mathrm{K}, \mathrm{Fe}, \mathrm{Ti}$ and $\mathrm{P}$ concentrations as the enriched intra-orogenic intrusions. The data have higher magnesium numbers compared to the syn-, post- and intraorogenic groups with the gabbro group forming the higher end group (Figs 5 and 6).

In addition to the gabbro group, plagioclase porphyrite and 36-MJV-08 samples stand out from the monzogabbro group. The plagioclase porphyrite sample has a fairly similar character as the gabbro group. In contrast, the sample 36-MJV-08 shows strong enrichment in $\mathrm{P}_{2} \mathrm{O}_{5}(2.74$ wt $\%)$ and low $\mathrm{SiO}_{2}(38.02 \mathrm{wt} \%)$ concentration (Fig. 6).

\subsection{Trace elements}

The key trace element concentrations of the Moisio data are shown in Fig. 7. The monzogabbro group shows fairly high concentrations of $\mathrm{Ba}, \mathrm{Sr}, \mathrm{F}$ and $\mathrm{Nb}$ compared to the gabbro group. Consequently, the two groups can be easily identified by the trace-element concentrations with the monzogabbro group forming the higher end group with following concentrations; $\mathrm{Ba}$ > 345 ppm, $\mathrm{Sr}>750 \mathrm{ppm}, \mathrm{F}$ > 2000 ppm and $\mathrm{Nb}>11 \mathrm{ppm}$. Additionally, one anomalous sample (36-MJV-08) is identified with $\mathrm{F}$ concentration of 7120 ppm. In contrast, the $\mathrm{Ni}$ and $\mathrm{Cr}$ concentrations of the monzogabbro group are low compared to the gabbro group. The $\mathrm{Ni}$ concentrations vary between 10 and $60 \mathrm{ppm}$ with one anomalously high sample value of $127 \mathrm{ppm}$ and the $\mathrm{Cr}$ concentrations are between 10 and 110 ppm with three anomalously high samples between 220 and 560 ppm. The gabbro group generally forms a separate group with slightly higher concentrations in $\mathrm{Ni}$ and $\mathrm{Cr}$ including the mentioned three anomalously high samples. The REE diagram of all the Moisio samples shows LREE enrichment (Fig. 8). In the primitive mantle normalized multi-element diagram the Moisio data show enrichment of LILEs and certain high field strength elements (HFSE). The monzogabbro group show higher LREE contents than the gabbro group and also show negative anomalies in $\mathrm{Nb}, \mathrm{Pb}$ and $\mathrm{Zr}$ (Fig. 9).

The trace-element data from the Moisio
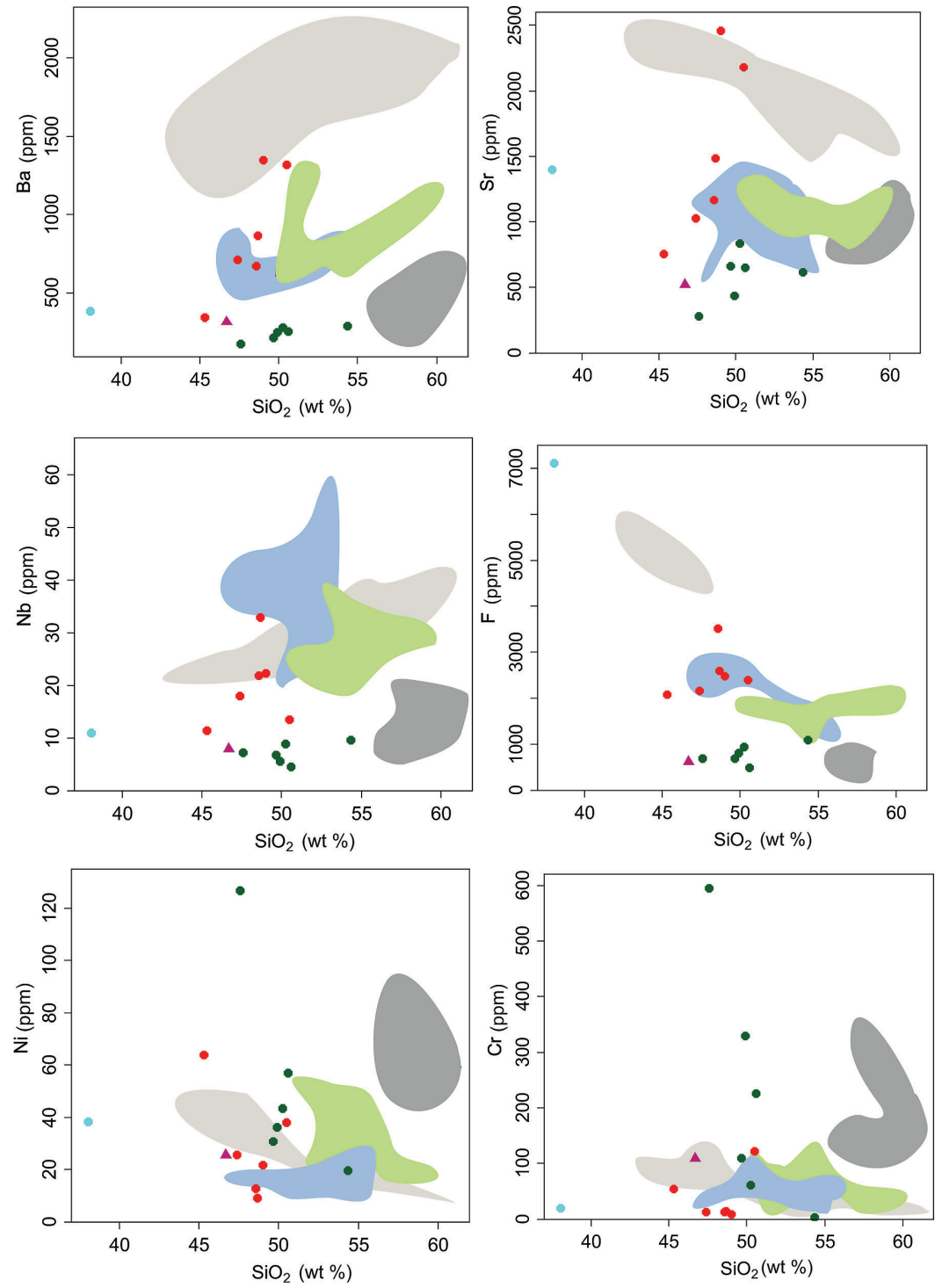

Fig. 7. Selected trace element diagrams. Symbols and fields as in Fig. 5. 
monzogabbro group resembles the intraorogenic reference data and plot between the syn- and post-orogenic reference fields.

\section{Discussion}

\subsection{Age data}

From the two investigated samples four apparently different age groups were obtained: $1859 \pm 5 \mathrm{Ma}$ for the Moisio sample and $1889 \pm 13 \mathrm{Ma}, 1863 \pm 17$ $\mathrm{Ma}$ and $1831 \pm 12 \mathrm{Ma}$ for the Turku sample. The age of the Moisio sample is inferred to be a crystallisation age of the magma on the basis of zircon morphology and consistency with field geology and other regional geochemical and geochronological information. Similar ages have been recorded in other plutonic rocks in SW Finland, e.g., diorites and gabbros in Olkiluoto, diorites in Rauma and Korpo and a charnockite in Lohja, all c. 1.86-1.85 Ga (Mänttäri et al., 2006, 2007; Pajunen, 2008; Väisänen et al., 2012b). Obviously, the c. $1.86 \mathrm{Ga}$ Moisio monzogabbro belongs to the same age group of mantle-derived intrusions which fall within the proposed intraorogenic stage.

The three ages obtained from the Turku sample are more complicated to interpret. The $1863 \pm 17 \mathrm{Ma}$ age is obtained both from the core and rim domains, whereas the 1831 $\pm 12 \mathrm{Ma}$ age was obtained from the rims. We interpret that the $1.86 \mathrm{Ga}$ age represents the same magma crystallisation event as in the other sample, i.e. the grains grew from the magma, also on the pre-existing zircons. The $1.83 \mathrm{Ga}$ is the approximate age of peak metamorphism in the area (Väisänen et al., 2002) and therefore we think that those rims grew during the metamorphism. We consider the $1.89 \mathrm{Ga}$ age to represent an inherited zircon population. Not only is the age c. 30 my older than the intrusion age, but also the zircon morphology is different. Many of the zircons are fragments and

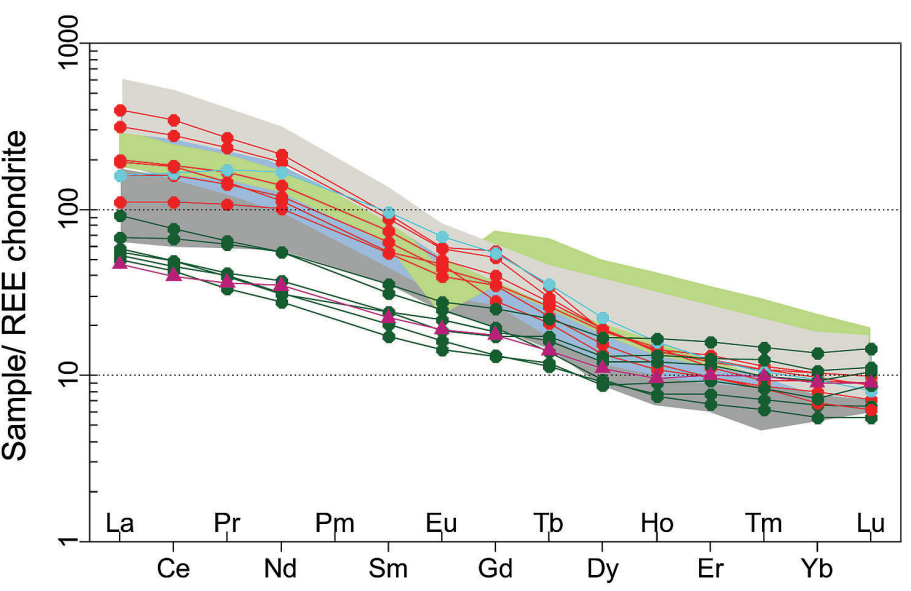

Fig. 8. The chondrite normalized REE diagram (normalization after Boynton, 1984). Symbols and fields as in Fig. 5.

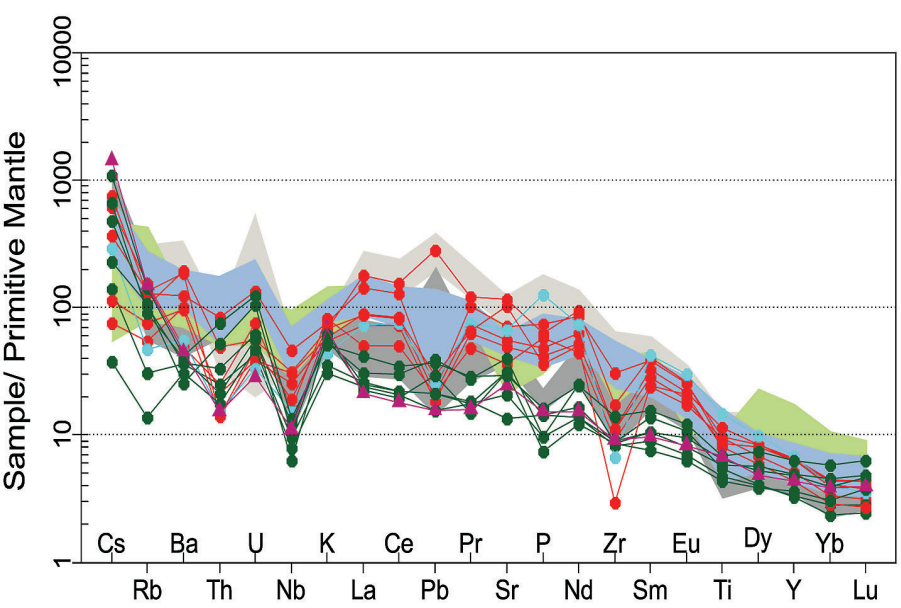

Fig. 9. The primitive mantle normalized multielement diagram (normalization after Sun \& McDonough, 1989). Symbols and fields as in Fig. 5.

show magmatic resorption features and new growth at the edges.

The origin of the inherited xenocrystic zircons is not quite clear. The age $1.89 \mathrm{Ga}$ is coeval with the preorogenic magmatism in which case the zircons could have crystallised in the mantle and remain trapped there until melting at $1.86 \mathrm{Ga}$ brought them up. Zircons grown in the mantle and then entrained in younger intrusions are not common, although described from kimberlites (e.g. Konzett et al. 1998, Peltonen \& Mänttäri, 2001) and some other mantle-derived rocks (Peltonen et al., 2003). The typical features of mantle zircons are, e.g., (i), large size, if intact, but normally the 
grains are broken to smaller fragments, (ii) varying zoning patterns and (iii) resorption features (Konzett et al., 1998; Corfu et al., 2003). Mantle zircons derive from fluid metasomatized parts where zircon growth is possible. These features are also common in our sample (Fig. 3), but not exclusive to mantle zircons. Therefore a crustal origin should be considered, too.

It is not likely that the xenocrystic zircons are of detrital origin. Firstly, the zircons are not abraded as would be expected for detrital zircons (Corfu et al., 2003). Secondly, earlier studies of detrital zircons in this part of the Svecofennian orogen have revealed ages older than c. $1.91 \mathrm{Ga}$, including Archaean ages (Lahtinen et al., 2002; Väisänen et al., 2002, Ehlers et al., 2004, Andersson et al., 2006b, Väisänen et al., 2012b). Therefore, assuming a crustal origin, the zircons derive from rocks belonging to the preorogenic stage of the Svecofennian orogeny. Further investigations combining detailed chemical and isotopic analysis of zircons would be required to decipher the source (c.f., Belousova et al., 2002, 2006).

\subsection{Geochemical data}

From the Moisio data two main geochemical groups can be identified; an enriched monzogabbro and a less enriched gabbro group. The monzogabbro group shows higher degree of enrichment in $\mathrm{K}, \mathrm{Fe}$, $\mathrm{P}, \mathrm{Ba}, \mathrm{Sr}, \mathrm{Zr}, \mathrm{F}, \mathrm{Nb}$ and LREE whereas the gabbro group has higher $\mathrm{Ni}$, and $\mathrm{Cr}$ concentrations (Figs 7-9). The differences between the two groups imply different magma sources with the gabbro group originating from a more primitive one. Comparison of the Moisio data with the syn- intra- and postorogenic data shows that the monzogabbro group closely resembles the intra-orogenic Rauma and Korpo intrusions whereas the gabbro-gabbroic diorite group shows closer similarities with the synorogenic Uusikaupunki data.

The monzogabbro group has clearly an enriched magma source showing high LREE, LILE, $\mathrm{P}$ and $\mathrm{F}$ concentrations suggesting a subduction related enrichment in the magma source (Pearce, 1982; Eklund et al., 1998). The negative $\mathrm{Nb}, \mathrm{Pb}$ and $\mathrm{Zr}$ anomalies indicate enrichment of the adjacent relatively more mobile elements rather than depletion of these elements (Fig. 9). The $\mathrm{P}_{2} \mathrm{O}_{5} / \mathrm{TiO}_{2}$ ratio is used to estimate lithospheric mantle carbonate metasomatism (Baker \& Wyllie, 1992). Fig. 10 shows high $\mathrm{P}_{2} \mathrm{O}_{5} / \mathrm{TiO}_{2}$ ratios in the monzogabbro group implies carbonate metasomatism in the source, (c.f. Eklund et al., 1998 and Andersson et al., 2006a). The two highly mobile elements $\mathrm{Ba}+\mathrm{Sr}$ plotted against less mobile La (Fig. 11) indicate intensive subduction-related mantle metasomatism within the monzogabbro group. The strong relative enrichment of LREEs and $\mathrm{Sr}$ over HREEs and HFSEs further supports possible carbonate metasomatism (Ionov et al., 1993), which is identifiable in the monzogabbro group of the Moisio data. In contrast, the gabbro group does not show the same geochemical features and therefore represents a separate magmatic event (Fig. 9).

The two samples, which stand out from the main groups, are the plagioclase porphyrite sample and the 36-MJV-08 sample. Based on its geochemical composition, the plagioclase porphyrite sample is interpreted as a dyke related to the emplacement of the gabbro group. In contrast, the sample 36-MJV-08 shows very strong $\mathrm{P}_{2} \mathrm{O}_{5}(2.74$ wt \%) and $\mathrm{F}$ (7120 ppm) enrichment with low $\mathrm{SiO} 2$ $(<40 \mathrm{wt} \%)$ content. Apatite is very abundant and appears as major mineral. This suggests a cumulate character, similar to cumulates described from the postorogenic intrusions (Eklund et al., 1998; Rutanen et al. 2011).

Overall, the geochemical data of the monzogabbro group resemble the other intra-orogenic intrusions in SW Finland (Korpo and Rauma) whereas the gabbro group has closer similarities with the synorogenic intrusions (Uusikaupunki).

\subsection{Implications for the southern Svecofennian crustal evolution}

The age of the Moisio monzogabbro intrusion, $\sim 1.86 \mathrm{Ga}$, falls in with the proposed intra-orogenic $\sim 1.87-1.84$ Ga phase between the Fennian and Svecobaltic orogenies. Consequently, the Moisio monzogabbro provides additional evidence of 
mantle-derived magmatism during the intraorogenic period. Recently, metamorphic zircons as old as 1.84-1.85 Ga have been dated (Torvela et al., 2008; Väisänen et al., 2012b). Similarly, anatectic granites of similar age have been dated, such as the $1852 \pm 3 \mathrm{Ma}$ Veikkola granite (Kurhila et al., 2005), the $1849 \pm 8 \mathrm{Ma}$ Korpo granite (Väisänen et al., 2012a), and the $1843 \pm 3 \mathrm{Ma}$ Västern-

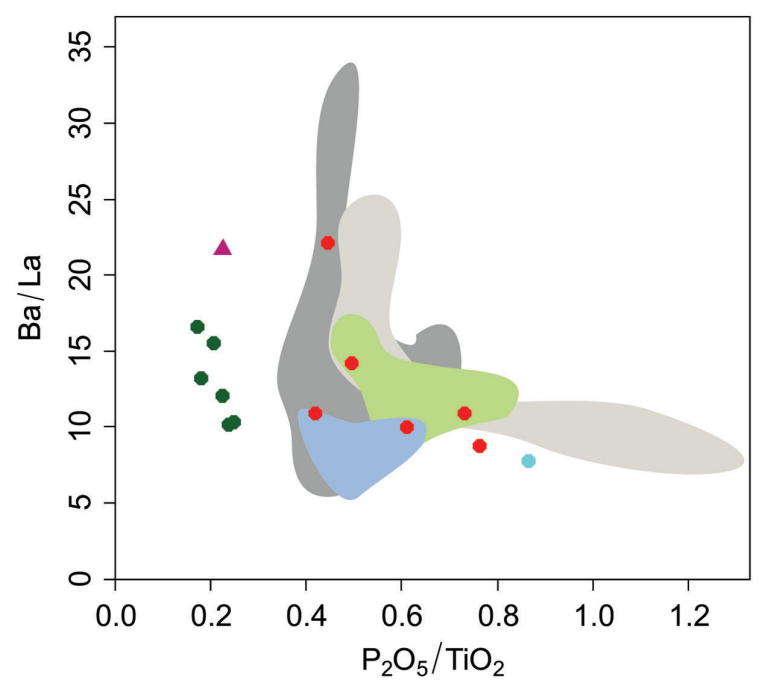

Fig. 10. $\mathrm{Ba} / \mathrm{La}$ vs $\mathrm{P}_{2} \mathrm{O}_{5} / \mathrm{TiO}_{2}$ shows variation between more fluid enriched source (high $\mathrm{Ba} / \mathrm{La}$ ) and more carbonate metasomatism affected source (high $\mathrm{P}_{2} \mathrm{O}_{5} / \mathrm{TiO}_{2}$ ) after Baker \& Wyllie (1992). Symbols and fields as in Fig. 5.

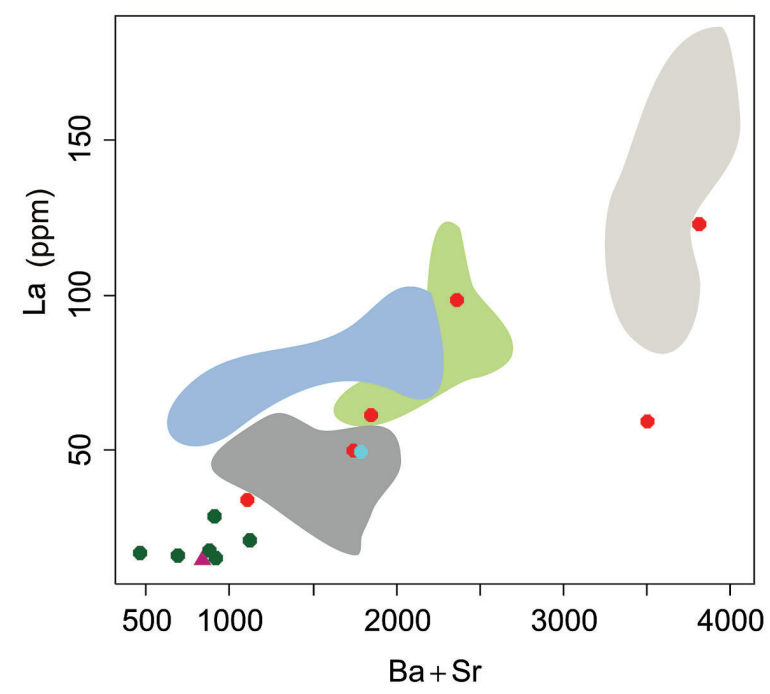

Fig. 11. La vs Ba+Sr diagram. Higher concentrations indicate greater degree of the enrichment in the source. Symbols and fields as in Fig. 5. kvarn granite (Skyttä \& Mänttäri, 2008). These observations indicate that the onset of the high heat flow, which culminated at c. $1825 \mathrm{Ma}$ in high grade metamorphism and crustal melting, was initiated already during the intra-orogenic stage. The Moisio monzogabbro and other mantle derived intraorogenic intrusions (c.f. Väisänen et al., 2012a) combined with radioactive decay within granitoids (Kukkonen \& Lauri, 2009) probably provided the heat necessary for the process.

The geotectonic environment of the intraorogenic phase is ambiguous. Evidently, southern Svecofennia experienced extension during the intraorogenic period with simultaneous mantle-derived magmatism in the deeper part and sedimentary basin formation in the upper part of the crust. The driving force for the extension is still debatable whether it is due to a collapse of the Fennian orogen (Korja et al., 2006), far-field extension during outboard subduction (Lahtinen \& Nironen, 2010) or fluctuating stresses between convergence and divergence caused by a retreating subduction zone (Hermansson et al., 2008; Saalmann et al., 2009). If the extension was caused by a retreating subduction zone, it probably was not a single event but a series of several retreating extensional phases alternating with compressional advancing ones (Hermansson et al., 2008). The subductiontriggered extension is a tempting model from another point of view. According to Condie (2013) the Svecofennian orogeny is characterized by longlived subduction that overlasted the different orogenic phases. Subduction erosion and sediment subduction introduce extensive amounts of continental material and water into the mantle wedge (e.g. Cawood et al., 2009). This would effectively enrich the mantle wedge with incompatible elements, which during subsequent melting would produce enriched mafic magmas. The amount of enrichment would increase with time as more and more continental material is introduced into the mantle wedge. The available data from southern Svecofennia show that enrichment of mafic mantle-derived rocks increases with time from preorogenic to postorogenic phases (Väisänen et al., 2012a; this study). 


\section{Conclusion}

Three different ages were obtained from the two studied samples. The zircons in the Moisio sample are prismatic crystals, whereas the Turku sample mostly consists of zircon fragments and resorbed crystals. The $\sim 1.86 \mathrm{Ga}$ age of the Moisio sample reflects the age of the intrusion whereas the age of the Turku sample $\sim 1.89$ Ga represents the age of an inherited zircon population, entrained either from the mantle or the preorogenic Svecofennian crust. Additionally, $\sim 1.83$ Ga zircon rim domains reflect the metamorphic growth. The $\sim 1.86 \mathrm{Ga}$ age coincides with the intra-orogenic 1.87-1.84 Ga extensional period in southern Finland.

The geochemical study of the Moisio mafic rocks distinguished two main groups; an enriched monzogabbro group and a gabbro group. The monzogabbro group shows high-K to shoshonitic compositions and distinctively high LREE, LILE, Fe-, P-, Ti- and F-concentrations. The magma source is likely to have experienced subduction related carbonate metasomatism, induced by sediment subduction and/or subduction erosion. The Moisio monzogabbro group resembles in composition the intra-orogenic Rauma and Korpo intrusions. The gabbro group does not show similar enrichment and is therefore considered a separate magmatic event which shows a closer kinship with the synorogenic igneous rocks in the area.

Evidently, the Moisio monzogabbro intrusion represents enriched, mantle-derived magmatism from the intra-orogenic period. It provides additional data to support the recent model for the role of the mantle in the thermal evolution of the crust in southern Svecofennia.

\section{Acknowledgements}

The reviews by Laura Lauri, Mikko Nironen and the Editor Jussi Heinonen are highly appreciated in improving the manuscript. Arto Peltola made the zircon mounts and Sari Niemi helped with the thin section making. The study was funded by the Otto A. Malm Foundation and the Finnish Cultural Foundation, Varsinais-Suomi Regional Fund. This is a Finnish Isotope Geosciences Laboratory contribution.

\section{References}

Andersson, U.B., Eklund, O., Fröjdö, S. \& Konopelko, D. 2006a. 1.8 Ga magmatism in the Fennoscandian Shield; lateral variations in subcontinental mantle enrichment. Lithos 86, 110-136.

Andersson, U.B., Högdahl, K., Sjöström, H. \& Bergman, S. 2006b. Multistage growth and reworking of the Palaeoproterozoic crust in the Bergslagen area, southern Sweden: evidence from U-Pb geochronology. Geological Magazine 143, 679-697.

Bedrock of Finland - DigiKP. Digital map database [Electronic resource]. Geological Survey of Finland, Espoo. Version 1.0. [referred 05.02.2014]

Baker, M.B. \& Wyllie, P.J. 1992. High-pressure apatite solubility in carbonate-rich liquids: Implications for mantle metasomatism. Geochimica et Cosmochimica Acta 56, 3409-3422.

Belousova, E.A., Griffin, W.L., O’Reilly, S.Y. \& Fisher, N.I. 2002. Igneous zircon: trace-element composition as an indicator of source rock type. Contributions to Mineralogy and Petrology 143, 602-622.

Belousova, E.A., Griffin, W.L. \& O'Reilly, S.Y. 2006. Zircon Crystal Morphology, Trace Element Signatures and Hf Isotope Composition as a Tool for Petrogenetic Modelling: Examples From Eastern Australian Granitoids. Journal of Petrology 47, 329-353.

Bergman, S., Högdahl, K., Nironen, M., Ogenhall, E., Sjöström, H., Lundqvist, L. \& Lahtinen, R. 2008. Timing of Palaeoproterozoic intra-orogenic sedimentation in the central Fennoscandian Shield; evidence from detrital zircon metasandstone. Precambrian Research 161, 231249.

Boynton, W.V. 1984. Cosmochemistry of the rare earth elements: meteoric studies. Rare earth element geochemistry, 63-114.

Cawood, P.A., Kröner, A., Collins, W.J., Kusky, T.M., Mooney, W.D., \& Windley, B.F. 2009. Accretionary orogens through Earth history. Geological Society, London, Special Publications 318, 1-36.

Claesson, S., Huhma, H., Kinny, P.D. \& Williams, I.S. 1993. Svecofennian detrital zircon ages-implications for the Precambrian evolution of the Baltic Shield. Precambrian Research 64, 109-130.

Condie, K.C. 2013. Preservation and recycling of crust during accretionary and collisional phases of Proterozoic orogens: a bumpy road from Nuna to Rodinia. Geosciences 3, 240 261.

Corfu, F., Hanchar, J.M., Hoskin, P.W. \& Kinny, P. 2003. Atlas of Zircon Textures. Reviews in Mineralogy and Geochemistry 53, 469-500.

van Duin, J.A. 1992. The Turku granulite area, SW Finland: a fluid-absent Svecofennian granulite occurrence. PhDthesis, Vrije Universiteit te Amsterdam, 234 pp. 
Ehlers, C., Lindroos, A. \& Selonen, O. 1993. The late Svecofennian granite-migmatite zone of southern Finland - a belt of transpressive deformation and granite emplacement. Precambrian Research 64, 295-309.

Ehlers, C., Skiöld, T. \& Vaasjoki, M. 2004. Timing of Svecofennian crustal growth and collisional tectonics in Åland, SW Finland. Bulletin of the Geological Society of Finland 76, 63-91.

Eklund, O., Konopelko, D., Rutanen, H., Fröjdö, S. \& Shebanov, A.D. 1998. 1.8 Ga Svecofennian postcollisional shoshonitic magmatism in the Fennoscandian shield. Lithos 45, 87-108.

Hermansson, T., Stephens, M.B., Corfu, F., Page, L.M. \& Andersson, J. 2008. Migratory tectonic switching, western Svecofennian orogen, central Sweden: Constraints from $\mathrm{U} / \mathrm{Pb}$ zircon and titanite geochronology. Precambrian Research 161, 250-278.

Högdahl, K., Sjöström, H., Andersson, U.B. \& Ahl, M. 2008. Continental margin magmatism and migmatisation in the west-central Fennoscandian Shield. Lithos 102, 435-459.

Horstwood, M.S.A., Foster, G.L., Parrish, R.R., Noble, S.R. \& Nowell, G.M. 2003. Common Pb-corrected in situ U$\mathrm{Pb}$ acessory mineral geochronology by LA-MCICP-MS. Journal of Analytical Atomic Spectrometry 18, 837-846.

Huhma, H., Mänttäri, I., Peltonen, P., Kontinen, A., Halkoaho, T., Hanski., E., Hokkanen. T., Hölttä, P., Juopperi, H., Konnunaho, J., Lahaye, Y., Luukkonen., E., Pietikäinen, K., Pulkkinen, A., Sorjonen-Ward, P., Vaasjoki, M. \& Whitehouse, M. 2012. The age of Archaean greenstone belts in Finland. In: Höltta, P. (Ed.) The Archaean of the Karelia Province in Finland. Espoo: Geological Survey of Finland, Special Paper 54, 73-174.

Ionov, D.A., Dupuy, C., O’Reilly, S.Y., Kopylova, M.G. \& Genshaft, Y.S. 1993. Carbonate peridotite xenoliths from Spitsbergen: implications for trace-element signature of mantle carbonate metasomatism. Earth and Planetary Science Letters 119, 283-297.

Janoušek, V., Farrow, C. M. \& Erban, V. 2006. Interpretation of Whole-rock Geochemical Data in Igneous Geochemistry: Introducing Geochemical Data Toolkit (GCDkit). Journal of Petrology 47, 1255-1259.

Johannes, W., Ehlers, C., Kriegsman, L.M. \& Mengel, K. 2003. The link between migmatites and S-type granites in the Turku area, southern Finland. Lithos 68, 69-60.

Kähkönen, Y. 2005. Svecofennian supracrustal rocks. In: Lehtinen M., Nurmi P. A. \& Rämö O. T. (Eds) Precambrian Geology of Finland - Key to the Evolution of the Fennoscandian Shield. Elsevier B.V., 343-406.

Konzett, J., Armstrong, R. A., Sweeney, R.J. \& Compston, W. 1998. The timing of MARID metasomatism in the Kaapvaal mantle: an ion probe study of zircons from MARID xenoliths. Earth and Planetary Science Letter 160, 133-145.

Korja, A., Lahtinen, R. \& Nironen, M. 2006. The Svecofennian orogen: a collage of microcontinents and island arcs. Geological Society of London, Memoirs 32, 561-578.

Kukkonen, I.T. \& Lauri, L.S. 2009. Modelling the thermal evolution of a collisional Precambrian orogen: High heat production migmatitic granites of southern Finland. Precambrian Research 168, 233-246.

Kurhila, M., Vaasjoki, M., Mänttäri, I., Rämö, T. \& Nironen, M. 2005. U-Pb ages and Nd isotope characteristics of the lateorogenic migmatizing microline granites in southwestren Finland. Bulletin of the Geological Society of Finland 77, 105-128.

Kurhila, M., Andersen, T. \& Rämö, O.T. 2010. Diverse sources of crustal granitic magma: Lu-Hf isotope data on zircon in three Palaeoproterozoic leucogranites of southern Finland. Lithos 115, 263-271.

Lahtinen, R. \& Nironen, M. 2010. Palaeoproterozoic lateritic paleosol-ultramature/mature quartzite-meta-arkose successions in Southern Fennoscandia - intraorogenic stage during the Svecofennian orogeny. Precambrian Research 183, 770-790.

Lahtinen, R., Huhma, H. \& Kousa, J. 2002. Contrasting source components of the Palaeoproterozoic Svecofennian metasediments: Detrital zircon U-Pb, Sm-Nd and geochemical data. Precambrian Research 116, 81-109

Lahtinen, R., Korja, A. \& Nironen, M. 2005. Palaeoproterozoic tectonic evolution. In: Lehtinen, M., Nurmi, P. A. \& Rämö, O. T. (eds) Precambrian Geology of Finland - Key to the Fennoscandian Shield. Elsevier B.V., 481-532.

Ludwig, K.R. 2003. Isoplot/Ex, version 3. A geochronological toolkit for Microsoft excel: Berkeley. California, Geochronology Center. Special Publication No. 4, 70 pp.

Mänttäri, I., Talikka, M., Paulamäki, S. \& Mattila, J. 2006. U$\mathrm{Pb}$ Ages of Tonalitic Gneiss, Pegmatite Granite, and Diabase Dyke, Olkiluoto Study Site, Eurajoki, SW Finland. Posiva Working Report 2006-12, 18 pp.

Mänttäri, I., Aaltonen, I. \& Lindberg, A. 2007. U-Pb Ages for Two Tonalitic Gneisses, Pegmatite Granites, and K-feldspar Porphyries, Olkiluoto Study Site, Eurajoki, SW Finland. Posiva Working Report 2007-70, 44 pp.

Middlemost, E.A.K. 1985. Magmas and magmatic rocks. London: Longman, $266 \mathrm{pp}$.

Mouri, H., Väisänen, M., Huhma, H. \& Korsman, K. 2005. $\mathrm{Sm}-\mathrm{Nd}$ garnet and $\mathrm{U}-\mathrm{Pb}$ monazite dating of high-grade metamorphism and crustal melting in the West Uusimaa area, southern Finland. GFF 127, 123-128.

Nevalainen, J., 2014. Single-grain zircon U-Pb dating and geochemistry of the Moisio monzogabbro, Turku, SW Finland. M.Sc. Thesis. University of Turku, 55 p.

Nironen, M. 2005. Proterozoic orogenic granitoid rocks. In: Lehtinen M., Nurmi P. A. \& Rämö O. T., (eds) Precambrian Geology of Finland - Key to the Evolution of the Fennoscandian Shield. Elsevier B.V., 443-480.

Nironen, M. 2011. The Pyhäntaka formation, southern Finland: A sequence of metasandstones and metavolcanic 
rocks upon an intra-orogenic unconformity. Bulletin of the Geological Society of Finland 83, 5-23.

Nironen, M. \& Mänttäri, I. 2012. Timing of accretion, intraorogenic sedimentation and basin inversion in the Palaeoproterozoic Svecofennian orogen: The Pyhäntaka area, southern Finland. Precambrian Research 192, 34-51.

Pajunen, M. Airo, M-L, Elminen, T., Mänttäri, I., Niemelä, R., Vaarma, M., Wasenius, P. \& Wennerström, M. 2008. Tectonic evolution of the Svecofennian crust in Southern Finland. Geological Survey of Finland, Special Paper 47, $15-160$

Patchett, J. \& Kouvo, O. 1986. Origin of continental crust of 1.9-1.7 Ga age: $\mathrm{Nd}$ isotopes and $\mathrm{U}-\mathrm{Pb}$ zircon ages in the Svecokarelian terrain of South Finland. Contributions to Mineralogy and Petrology 92, 1-12.

Pearce, J.A. 1982. Trace element characteristics of lavas from destructive plate boundaries. In: Thorpe, R. S. (Ed.). Andesites. Chichester: Wiley, 528-548.

Peccerillo, R. \& Taylor, S.R. 1976. Geochemistry of Eocene calc-alkaline volcanic rocks from the Kastamonu area, northern Turkey. Contributions to Mineralogy and Petrology 58, 63-81.

Peltonen, P. \& Mänttari, I. 2001. An ion microprobe U-Th$\mathrm{Pb}$ study of zircon xenocrysts from the Lahtojoki kimberlite pipe, eastern Finland. Bulletin of the Geological Society of Finland 73, 47-58.

Peltonen, P., Mänttäri, I., Huhma, H. \& Konttinen, A. 2003. Archaean zircons from the mantle: The Jormua ophiolite revisited. Geology 31, 645-648.

Rosa, D.R.N., Finch, A.A., Andersen, T. \& Inverno, C.M.C. 2009. U-Pb geochronology and $\mathrm{Hf}$ isotope ratios of magmatic zircons from the Iberian Pyrite Belt. Mineralogy and Petrology 95, 47-69.

Rutanen, H., Andersson, U.B., Väisänen, M., Johansson, Å., Fröjdö, S., Lahaye, Y. \& Eklund, O. 2011. 1.8 Ga magmatism in Southern Finland: strongly enriched mantle and juvenile crustal sources in post-collisional setting. International Geology Review 53, 1622-1683.

Saalmann, K., Mänttäri, I., Ruffet, G. \& Whitehouse, M.J. 2009. Age and tectonic framework of structurally controlled Palaeoproterozoic gold mineralization in the Häme belt of southern Finland. Precambrian Research 174, 53-77.

Simonen, A. 1980. The Precambrian in Finland. Geological Survey of Finland, Bulletin 304, 1-58.

Skyttä, P. \& Mänttäri, I. 2008. Structural setting of late Svecofennian granites and pegmatites in Uusimaa Belt, SW Finland: Age constraints and implications for crustal evolution. Precambrian Research 164, 86-109.

Stacey, J.S. \& Kramers, J.D. 1975. Approximation of terrestrial $\mathrm{Pb}$ isotope evolution by a two-stage model. Earth and Planetary Science Letters 26, 207-221.

Sun, S.-S. \& McDonough, W.F. 1989. Chemical and isotopic systematics of oceanic basalts: Implications for mantle composition and processes. Geological Society, London, Special Publications 42, 313-345.

Suominen, V. 1991. The chronostratigraphy of southwestern Finland with special reference to Postjotnian and subjotnian diabases. Geological Survey of Finland, Bulletin 356, 100 pp.

Torvela, T., Mänttäri, I. \& Hermansson, T. 2008. Timing of deformation phases within the South Finland shear zone, SW Finland. Precambrian Research 160, 277-298.

Väisänen, M. 2004. Mynämäen karttalehden 1044 kallioperäkartoituksen loppuraportti. Geological Survey of Finland, Archive Report K 21.42/2004/2, 22 pp. (in Finnish)

Väisänen, M. \& Hölttä, P. 1999. Structural and metamorphic evolution of the Turku migmatite complex, southwestern Finland. Bulletin of Geological Society of Finland 71, 177218.

Väisänen, M. \& Kirkland, C.L. 2008. U-Th-Pb zircon geochronology on igneous rocks in the Toija and Salittu Formations, Orijärvi area, southwestern Finland: constraints on the age of volcanism and metamorphism. Bulletin of the Geological Society of Finland 80, 73-87.

Väisänen, M., Mänttäri, I., Kriegsman, L.M. \& Hölttä, P. 2000. Tectonic setting of post-collisional magmatism in the Palaeoproterozoic Svecofennian Orogen, SW Finland. Lithos 54, 63-81.

Väisänen, M., Mänttäri, I. \& Hölttä, P. 2002. Svecofennian magmatism and metamorphic evolution in southwestern Finland as revealed by $\mathrm{U}-\mathrm{Pb}$ zircon SIMS geochronology. Precambrian Research 116, 111-127.

Väisänen, M., Eklund, O., Lahaye, Y., O’Brien, H., Fröjdö, S., Högdahl, K. \& Lammi, M. 2012a. Intra-orogenic Svecofennian magmatism in SW Finland constrained by LA-MC-ICP-MS zircon dating and geochemistry. GFF 134, 99-114.

Väisänen, M., Johansson, Å., Andersson, U.B., Eklund, O. \& Hölttä, P. 2012b. Palaeoproterozoic adakite- and TTGlike magmatism in the Svecofennian orogen, SW Finland. Geologica Acta 10, 1-20.

Woodard, J. \& Hetherington, C.J. 2014. Carbonatite in a postcollisional tectonic setting: Geochronology and emplacement conditions at Naantali, SW Finland. Precambrian Research 240, 94-107. 